\title{
THE CHARACTERIZATION OF PLANE COLLINEATIONS IN TERMS OF HOMOLOGOUS FAMILIES OF LINES*
}

\author{
BY \\ WALTER PRENOWITZ
}

Introduction. This paper is concerned with the problem of specifying minimal conditions that a transformation be a plane collineation. $\dagger$

Let $\Gamma$ represent any region $\ddagger$ of the euclidean plane. A set of line intervals $\S$ contained in $\Gamma$ is called a family of lines or a family of lines in $\Gamma$, if the end points of each interval of the set are not in $\Gamma$, and each point of $\Gamma$ is on exactly one interval of the set. The region $\Gamma$ is said to contain the family of lines. If $n$ families of lines are contained in $\Gamma$, and no two have a common line, they are said to constitute an $n$-web of lines in $\Gamma$.

We may now state our principal result.

THEOREM V. Any topological transformation of region $\Gamma$ which carries a 4-web of lines in $\Gamma$ into a 4-web of lines is a projective collineation.

This theorem was proved by E. Kasner|| on the assumption that the transformation is differentiable twice. Other results characterizing projective transformations have been derived by $\mathrm{E}$. Gourin $\uparrow$ and by $\mathrm{W}$. Blaschke and his co-workers at Hamburg in connection with their study of webs of curves. ${ }^{* *}$ The theorem is related to the work of the Hamburg geometers on transformations of webs of curves since it is equivalent to the assertion that if two 4-webs of lines are topologically equivalent, thev are projectively equivalent.

The proof of this theorem is given in $\$ I I I$ of the paper. It is preceded by a set of lemmas in $\S I I$, which establish the requisite properties of families and

* Presented to the Society, March 26, 1932, and October 27, 1934; received by the editors January 20, 1935.

$\dagger$ We use the term collineation in the sense of a one-to-one transformation of a point set which carries collinear points into collinear points. Any projective transformation which carries points into points is a collineation.

$\ddagger$ The word region is used in the sense of an open set of points, such that any two points of the set can be joined by a broken line wholly contained in the set.

$\S$ The term line interval is used in the general sense to include half lines and infinite lines as well as finite intervals.

|| Bulletin of the American Mathematical Society, vol. 9 (1903), pp. 545-546.

T.In an unpublished manuscript he proved that any one-to-one transformation on the general projective plane, which carries four independent pencils of lines into four pencils of lines, carries a net of rationality into a net of rationality. This result first attracted the writer to the present problem.

** See Mayrhofer, Mathematische Zeitschrift, vol. 28 (1928), p. 733; Reidemeister, ibid., vol. 29 (1929), p. 433; Mayrhofer, ibid., vol. 30 (1929), p. 142, and Abhandlungen Hamburg Seminar, vol. 7 (1929), pp. 9, 10; Blaschke, Abhandlungen Hamburg Seminar, vol. 7, p. 69; Podehl, ibid., vol. 7, p. 397. 
webs of lines, and of transformations which carry webs of lines into webs of lines. The most important of these lemmas are the following:

LEMMA X. If a topological transformation of region $\Gamma$ carries a regular* 3-web of lines in $\Gamma$ into a 3-web of lines, it is differentiable and its Jacobian is nowhere zero.

LEMмa XI. If a topological transformation $T$ carries w, a 3-web of lines, into a 3-web of lines, and a family of $w$ is not regular* at one of its lines, then $T$ is a projectivity on this line.

LEMмA XII. The slope function of a regular* family of lines is differentiable.

The proof of the main theorem consists of two parts in which different methods are used. If the given 4 -web contains a regular 3 -web we show that the transformation can be extended twice by the application of Lemmas $\mathrm{X}$ and XII. It is then easy to prove that the transformation is projective in the neighborhood of a point by a method essentially that of Kasner's paper. On the other hand, if the given 4-web does not contain a regular 3-web, Lemma $\mathrm{XI}$ makes it possible to show that the transformation is projective in the neighborhood of a point, without reference to questions of differentiability. In either case, the result then follows by Theorem IV, which asserts that any one-to-one transformation of region $\Gamma$ which carries a 3-web of lines in $\Gamma$ into a 3-web of lines, is projective, if it is projective in the neighborhood of one point.

In $\$ I$ we derive characterizations of collineations on the projective plane in terms of homologous pencils of lines, with and without the assumption of the continuity of the transformation. Likewise \$III contains characterizations of projective transformations of a region of the euclidean plane in terms of pencils rather than arbitrary families of lines, without the assumption of continuity.

\section{Collineations oN the PROJEctive Plane}

We assume only the postulates of alignment and extension, and the fundamental theorem of projective geometry $\dagger$ in the following theorem.

Theorem I. A one-to-one point transformation on the projective plane is a collineation, if it carries three independent $\ddagger$ pencils and a line not of these pencils into three pencils and a line respectively.

\footnotetext{
* The sense in which we use this term is given on p. 579.

$\dagger$ See Veblen and Young, Projective Geometry, vol. I, assumptions A, E, P.

$\ddagger$ Three pencils are called independent, if their vertices are not collinear, otherwise they are dependent.
} 
Let $A, B, C$ (Figure 1) be the vertices of the three given pencils, and $l$ the line which is not in the pencils. Let $D, E$ be the intersections of $l$ with $A B, A C$ respectively, and $F$ the intersection of $B E$ and $C D$. Let $A^{\prime}, B^{\prime}, C^{\prime}, F^{\prime}$ be the images of $A, B, C, F$ respectively, under the given transformation $T$. Then no three of the points $A^{\prime}, B^{\prime}, C^{\prime}, F^{\prime}$ are collinear and there is a projective collineation, $S$, which carries $A^{\prime}, B^{\prime}, C^{\prime}, F^{\prime}$ into $A, B, C, F$ respectively. Let $R$ be the resultant of $T$ and $S$. Transformation $R$ preserves points $A, B$, $C, D, E, F$, pencils $A, B, C$, and line $l$.

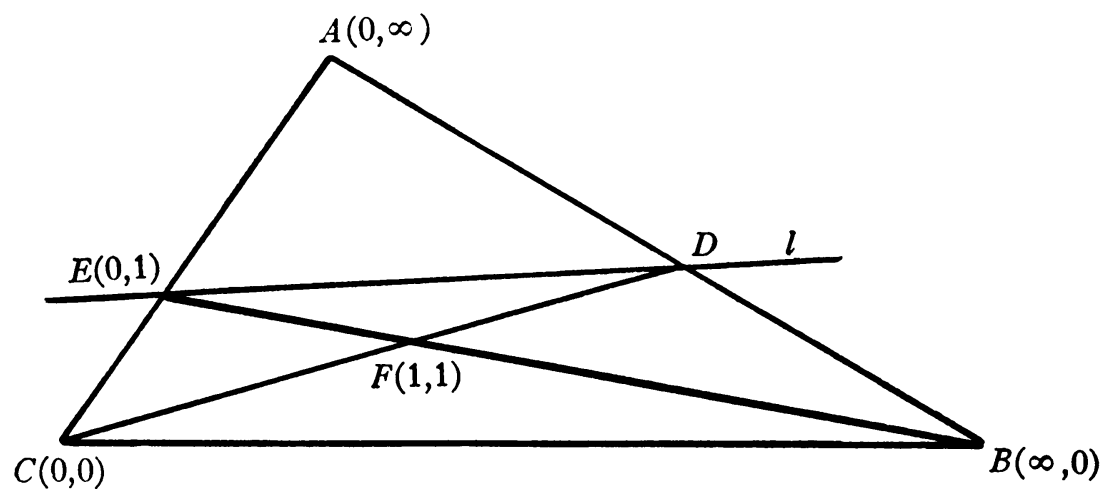

FIGURE 1

Now we introduce non-homogeneous coordinates in the plane so that $A, B, C, F$ are designated by $(0, \infty),(\infty, 0),(0,0),(1,1)$ respectively. Then any point, not on the line at infinity, is representable by $(x, y)$ where $x$ and $y$ are elements of some field.

Thus, $R$ is represented by

$$
x^{\prime}=\phi(x, y), \quad y^{\prime}=\psi(x, y),
$$

and pencils $A, B, C$ by the equations $x=t, y=t, y=t x$ respectively, where $t$ is a variable element of the field. The invariance of the pencils $x=t, y=t \mathrm{im}-$ plies that $\phi$ is independent of $y$ and $\psi$ of $x$. Hence $R$ may be expressed by the equations

$$
x^{\prime}=\phi(x), \quad y^{\prime}=\psi(y) .
$$

Moreover, since the line $y=x$ is invariant, $R$ may be represented in the more simple form

$$
x^{\prime}=\phi(x), \quad y^{\prime}=\phi(y) .
$$

The invariance of the pencil $y=t x$ yields

$$
\phi(t x)=p(t) \phi(x) .
$$


Since $R$ leaves $(0,0)$ and $(1,1)$ invariant, $\phi(0)=0$ and $\phi(1)=1$. Substituting $x=1$ in (1) and eliminating $p(t)$ from (1), we have

$$
\phi(t x)=\phi(t) \phi(x) .
$$

Since the equation of line $l$ is $y=x+1$, its invariance implies

$$
\phi(x+1)=\phi(x)+1 \text {. }
$$

We have from (2) and (3),

$$
\phi[t(y+1)]=\phi(t) \phi(y)+\phi(t)
$$

and

$$
\phi(t y+t)=\phi(t y)+\phi(t) .
$$

Substituting $x$ for $t y$ in (4), we may assert

$$
\phi(x+t)=\phi(x)+\phi(t)
$$

for all $x, t$ in the field.

The relations (2) and (5) are sufficient to prove that $R$ is a collineation. For the finite points of any line not in pencil $A$ are represented by the equation $y=m x+n$. Then

$$
y^{\prime}=\phi(m x+n)=\phi(m) \phi(x)+\phi(n)=\phi(m) x^{\prime}+\phi(n),
$$

so that collinear points not on $A B$ have collinear images, and parallel lines have parallel images. From this it is easy to show that $R$ is a collineation and $T$ is likewise.

For the case of the real projective plane, we have the following result.

CoRollary. A one-to-one transformation on the real projective plane is a projective collineation, if it carries three independent pencils and a line not of these pencils into three pencils and a line respectively.

In this case, the functional equations (2) and (5)* hold for the real field and it is known that the only common solution is $\phi(x)=x$. This may easily be proved. From (2) we have

$$
\phi\left(x^{2}\right)=[\phi(x)]^{2}
$$

so that $\phi(x)>0$, if $x>0$. This implies, in virtue of (5), that $\phi(x)$ is monotonic. By means of iteration on (5), we can show that

$$
\phi(r x)=r \phi(x)
$$

* Darboux, Mathematische Annalen, vol. 17 (1880), pp. 55-61, derives essentially these equations, in proving the fundamental theorem of projective geometry. We use his method of solution. 
and hence that

$$
\phi(r)=r
$$

for all rational $r$. Since $\phi$ is monotonic, (6) holds for all real $r$. It easily follows that $R$ is the identical transformation. Thus $T$ is $S^{-1}$, a projective collineation.

The hypothesis of Theorem I cannot be lessened with respect to the additional line. For there exist transformations on the real projective plane which are not collineations and which carry three independent pencils of lines into three pencils. For example, the transformation

$$
x^{\prime}=x^{3}, \quad y^{\prime}=y^{3}, \quad t^{\prime}=t^{3}
$$

preserves the pencils $x=p t, y=p t, y=p x$, where $p$ is a parameter. The condition of independence of the three pencils also is essential. In fact, if $\phi(x)$ is a discontinuous solution of $(5)^{*}$ which assumes each real value exactly once, the equations

$$
\begin{array}{llll}
x^{\prime}=t \phi(x / t), & y^{\prime}=t \phi(y / t), & t^{\prime}=t & \\
x^{\prime}=x, & y^{\prime}=y, & t^{\prime}=t & (t=0),
\end{array}
$$

define a one-to-one transformation on the real projective plane which preserves the infinitude of pencils of the form $y=r x+p t$, where $r$ is rational and $p$ is a parameter.

However, if we assume that the transformation is continuous, we can lessen the remainder of the hypothesis a good deal. This is shown in the following theorem.

THEOREM II. A topological transformation $\dagger$ on the real projective plane is a projective collineation, if it carries into lines, the lines of two pencils with vertices $A, B$ and three additional lines which concur on $A B$, provided that these three lines and $A B$ are not in a net of rationality.

Let $C$ be the intersection of the three lines. Let $D$ be a point, distinct from $C$, on one of these lines, $E$ and $F$, the intersections of $A D$ with the other two lines, and $G$, the intersection of $C D$ and $B E$. (See Figure 2.) Then, as in the preceding theorem, apply to the given transformation a projective collineation, such that the resultant transformation leaves $A, B, D, G$ invariant. Introduce coordinates so that $A, B, D, G$ are represented by $(0, \infty),(\infty, 0)$, $(0,0),(1,1)$ respectively.

\footnotetext{
* See Hamel, Mathematische Annalen, vol. 60 (1905), p. 459.

$\dagger$ A topological transformation is a uniform continuous transformation which has a uniform continuous inverse.
} 


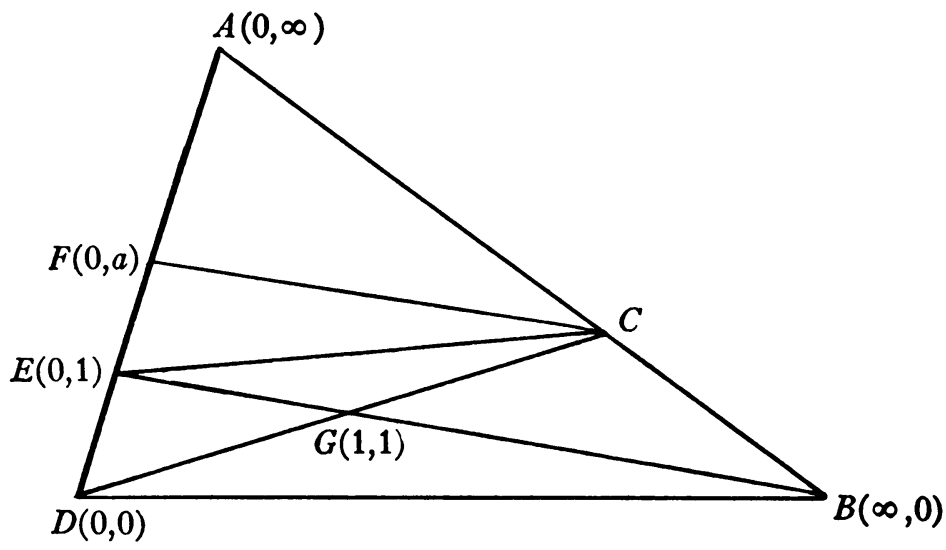

FIGURE 2

The equations of the three lines through $C$, and of their images, are, respectively, $y=x, y=x+1, y=x+a$ and $y=x, y=x+1, y=x+b$. Since, by hypothesis, $C F$ is not in the net of rationality determined by $C D, C E$ and $C A$, its intercept $a$, on the axis $D A$, is not in the domain of rationality determined by their intercepts on $D A$, namely $0,1, \infty$. Hence $a$ is an irrational number.

By the same argument as in the preceding theorem, the transformation takes the analytic form

$$
x^{\prime}=\phi(x), \quad y^{\prime}=\phi(y) .
$$

The functional equations characterizing $\phi$ are

$$
\phi(x+1)=\phi(x)+1
$$

and

$$
\phi(x+a)=\phi(x)+b .
$$

From these we get by iteration

$$
\phi(x+m+n a)=\phi(x)+m+n b
$$

where $m$ and $n$ are arbitrary integers.

Now if $x=0$ in (1), we have

$$
m+n b=\phi(m+n a),
$$

which with (1) yields

$$
\phi(x+m+n a)=\phi(x)+\phi(m+n a) .
$$


Since $a$ is irrational, $m$ and $n$ can be chosen so that $m+n a$ approximates any given real number as closely as we please. Thus,

$$
\phi(x+y)=\phi(x)+\phi(y)
$$

holds for all real $x$ and an everywhere dense set of values $y$. Hence (2) holds for all $x$ and $y$, since $\phi$ is continuous. Therefore $\phi(x)$ is of the form $c x$, which is the only continuous solution of the functional equation (2). In fact

$$
\phi(x)=x \text { since } c=\phi(1)=1 .
$$

From this the theorem follows as in the preceding corollary.

If the three lines mentioned in the hypothesis of the above theorem concur at a point not on $A B$, the result does not hold. This is evident from the first example given at the end of Theorem I.

If the three lines are not concurrent, the result holds with a slight modification as is shown in the following theorem.

THEOREM III. A topological transformation on the real projective plane is a projective collineation, if it carries into lines, the lines of two pencils $A, B$ and three additional non-concurrent lines, provided that no two of these three lines intersect on the line $A B$.

Let $C, D, E$ (Figure 3) be the intersections of pairs of the three non-concurrent lines. Consider $\triangle C D E$. It may be that the lines joining a vertex of $\triangle C D E$ to $A$ and $B$ are harmonically separated by the sides of $\triangle C D E$ which contain this vertex. But such an harmonic relationship cannot hold at each of two vertices of $\triangle C D E$. For suppose it does hold at $C$ and $D$. Then we have the harmonic sets of lines

$$
H[C A, C B ; C D, C E]
$$

and

$$
H[D A, D B ; D C, D E],
$$

three pairs of corresponding lines of which meet on $A B$. Hence the fourth pair, $C E, D E$, meet on $A B$. Thus, $E$ is on $A B$, contrary to the hypothesis.

Therefore, there is a vertex of $\triangle C D E$, such that the lines joining it to $A$ and $B$ are not harmonically separated by the sides of $\triangle C D E$ which contain this vertex. Let us suppose that $C$ is such a vertex.

Now, apply to the given transformation a projective collineation, such that the resultant transformation leaves $A, B, C, D$ invariant; and introduce coordinates so that $A, B, C, D$ are represented by $(0, \infty),(\infty, 0),(0,0),(1,1)$, respectively. 


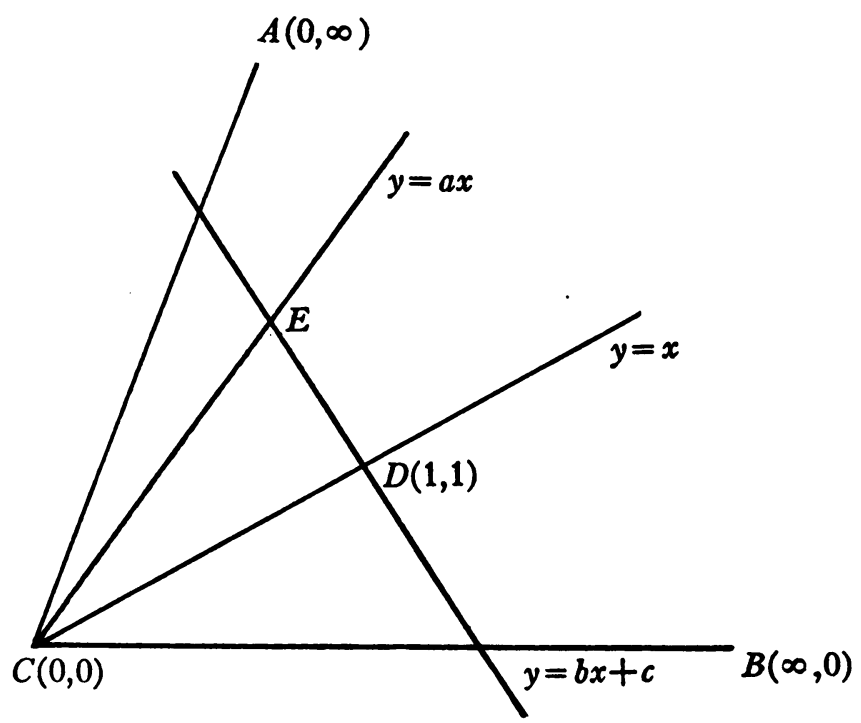

Figure 3

The equations of $C D, C E, D E$ are, respectively,

$$
\begin{aligned}
& y=x, \\
& y=a x \\
& y=b x+c
\end{aligned}
$$$$
(a \neq 0, \pm 1) \text {, }
$$$$
(b \neq 0, c \neq 0) \text {, }
$$

and those of their images are, respectively,

$$
\begin{aligned}
& y=x, \\
& y=d x \\
& y=e x+f
\end{aligned}
$$$$
\begin{array}{r}
(d \neq 0,1), \\
(e \neq 0, f \neq 0) .
\end{array}
$$

The transformation takes the form

$$
x^{\prime}=\phi(x), \quad y^{\prime}=\phi(y),
$$

where $\phi$ is characterized by the equations

$$
\begin{aligned}
\phi(a x) & =d \phi(x), \\
\phi(b x+c) & =e \phi(x)+f .
\end{aligned}
$$

We need only consider the case where $|a|>1$, since if $|a|<1$, we may replace (1) by

$$
\phi[(1 / a) x]=(1 / d) \phi(x)
$$

and proceed in the same manner. 
From (1) and (2), we have

$$
\phi(a b x+c)=\operatorname{de\phi }(x)+f
$$

which yields, in view of (3),

$$
\phi(b x+c / a)=e \phi(x)+f / d .
$$

By repetition of this procedure, we have

$$
\phi\left(b x+c / a^{m}\right)=e \phi(x)+f / d^{m}
$$

where $m$ is any positive integer. Let $m \rightarrow \infty$. Then, since $\phi$ is continuous, $f / d^{m}$ has a limit, which must be zero, as $d \neq 1$. Thus,

$$
\phi(b x)=e \phi(x),
$$

which, with (2), gives

$$
\phi(b x+c)=\phi(b x)+f
$$

and

$$
\phi(x+c)=\phi(x)+f .
$$

Thus

$$
\phi(x+n c)=\phi(x)+n f
$$

easily follows, where $n$ is any integer.

Applying to (5) the procedure used in deriving (4), we have

$$
\phi\left(x+n c / a^{p}\right)=\phi(x)+n f / d^{p},
$$

where $p$ is any non-negative integer. By iteration, (6) yields

$$
\begin{aligned}
& \phi\left[x+c\left(n_{0}+n_{1} / a^{1}+\cdots+n_{q} / a^{q}\right)\right] \\
& \quad=\phi(x)+f\left(n_{0}+n_{1} / d^{1}+\cdots+n_{q} / d^{q}\right)
\end{aligned}
$$

where the $n$ 's are arbitrary integers and $q$ is an arbitrary positive integer. Letting $x=0$ in (7), we may get

$$
\phi(x+y)=\phi(x)+\phi(y)
$$

where $x$ is any real number and

$$
y=c\left(n_{0}+n_{1} / a^{1}+\cdots+n_{q} / a^{q}\right) .
$$

Since we can choose $q$ and the $n$ 's so that $y$ approximates any given real number as closely as we please, (8) holds for all $x, y$ and the desired result follows as in the preceding theorem. 


\section{LEMMAS ON FAMILIES OF LINES}

We employ a series of lemmas on families and webs of lines, in proving the later theorems. Families of lines are indicated by the letters $f, g, h$, etc., and lines of a given family by the letter which represents the family, usually with subscripts affixed. Sometimes, the letter $f$ will be used to represent an arbitrary line of the family $f$, in which case the context will indicate the sense intended. Let family $f$ be contained in region $\Gamma$. Then if $P$ is any point of $\Gamma$, $f_{P}$ represents the line of the family $f$ which contains $P$. A line interval $l$, which is in $\Gamma$, and the end points of which are not in $\Gamma$, is called a transversal to $f_{P}$ at $Q$, if it intersects ${ }^{*} f_{P}$ at $Q$.

LEMMA I. If a transversal intersects $f_{P}$, it also intersects $f_{X}$, where $X$ is any point of some neighborhood of $P$.

Let $\Gamma$ be the region containing $f, l$ the transversal, and $Q$ the intersection of $l$ with $f_{P}$. Choose $A$ and $B$ on $f_{P}$, so that $P$ and $Q$ are between $A$ and $B$. (See Figure 4.)

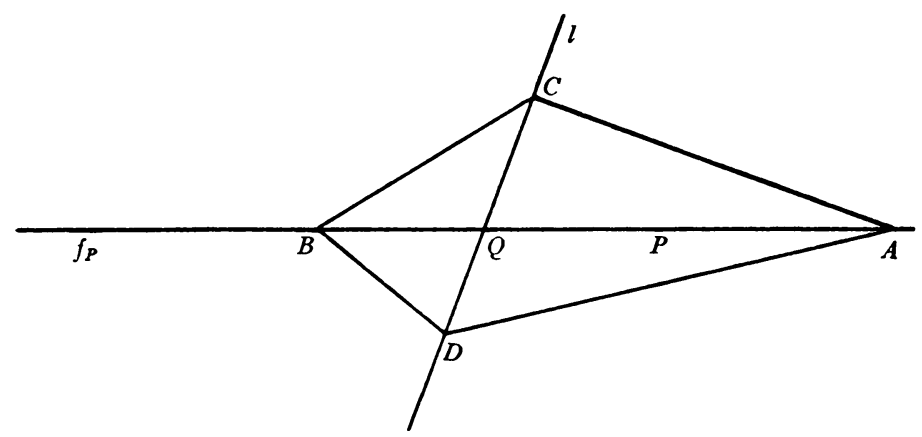

FIGURE 4

If $\Gamma$ has a boundary, the closed interval $A B$ has a positive distance $\epsilon$ from this boundary. Choose points $C$ and $D$ on $l$, so that $Q$ is between them and the distance of each from $Q$ is less than $\epsilon$. Then the quadrilateral $A C B D$ is contained in $\Gamma$, since the distance of each of its points from the closed interval $A B$ is less than $\epsilon$. If $X$ is any point inside the quadrilateral $A C B D, f_{X}$ intersects segment $\dagger C D$ and hence intersects $l$. Thus, since the interior of the quadrilateral $A C B D$ contains $P$, it may be taken as the required neighborhood and the lemma is proved.

COROLlARY. If $Q$ is a point of $f_{P}$ and $N$ is any neighborhood of $Q$, there exists $\bar{N}$, a neighborhood of $P$, such that if $X$ is in $\bar{N}, f_{X}$ contains a point of $N$.

* We say that lines $l$ and $m$ intersect, if they have exactly one common point.

$\dagger$ A segment is an open finite interval. 
Let $l$ be a transversal to $f_{P}$ at $Q$. Choose $A, B, C, D$ as in the lemma with the additional condition that segment $C D$ be in $N$. Then the interior of quadrilateral $A C B D$ can be chosen as $\bar{N}$.

DeFINITION. If in region $\Gamma$, containing a family of lines $f$, the sequence of points $\left\{P_{n}\right\}$ converges on point $P$, we say the sequence of lines $\left\{f_{P_{n}}\right\}$ approaches or converges on $f_{P}$, and we write $\left\{f_{P_{n}}\right\} \rightarrow f_{P}$.

LEMMA II. If $\left\{f_{n}\right\} \rightarrow f$ and $l$ is a transversal to $f$ at $Q$, then lintersects $\left\{f_{n}\right\}$ in a sequence of points $\left\{Q_{n}\right\}$ and $\left\{Q_{n}\right\} \rightarrow Q$.*

Since $\left\{f_{n}\right\} \rightarrow f$, there exists, for each $n$, a point $P_{n}$ on $f_{n}$ such that $\left\{P_{n}\right\} \rightarrow P$, where $P$ is some point on $f$. Thus, for all sufficiently large $n, l$ intersects $f_{n}$, by Lemma I, so that $\left\{Q_{n}\right\}$ exists.

Now, if we identify $P, Q$ and $l$ with the objects denoted by those symbols in Lemma I, we have that, for $C D$ any segment on $l$ containing $Q$, there exists $U$, a neighborhood of $P$, such that $f_{P_{n}}$ intersects $l$ in segment $C D$ for all $P_{n}$ contained in $U$. Thus, $Q_{n}$ lies in segment $C D$, any assigned neighborhood of $Q$, for all $n>p$, where $p$ is a natural number depending on the given neighborhood, and the lemma is proved.

DeFINITION. Let $l$ be a transversal to $f$, an arbitrary line of family $f$, and $l^{\prime}$, the infinite line which contains $l$. Let a fixed side of $l^{\prime}$ and a fixed direction on $l^{\prime}$ be specified. Then the inclination of $f$ with regard to $l$ is $\angle P Q R, \dagger$ where $Q$ is the intersection of $f$ and $l, P$ is a point of $f$ on the specified side of $l^{\prime}$, and $R$ is a point of $l$ in the specified direction from $Q$.

LEMMA III. The inclination of a line of a family with regard to a transversal is a continuous function of its intersection with the transversal.

Let the family be $f$ in region $\Gamma$ and the transversal $l$. Let $P_{1}, P$ (Figure 5) be, respectively, fixed and variable points of $l$, and $\theta_{1}, \theta$, the inclinations with regard to $l$ of $f_{P_{1}}, f_{P}$ respectively. We shall show that as $P \rightarrow P_{1}, \theta \rightarrow \theta_{1}$. If the extensions of $f_{P_{1}}, f_{P}$ outside of $\Gamma$ intersect, let the intersection be $Q$. Let the lengths $P P_{1}$ and $P Q$ be $s$ and $t$ respectively.

Then if $Q$ exists, we have

$$
\frac{\left|\sin \left(\theta-\theta_{1}\right)\right|}{\sin \theta_{1}}=\frac{s}{t},
$$

\footnotetext{
* We use the lemma frequently to infer convergence of a sequence of points from that of a sequence of lines. It is interesting to note that it guarantees that a convergent sequence of lines converges on a unique line.

† We use this symbol in two senses, viz., the angle $P Q R$ and the measure of the angle $P Q R$.
} 


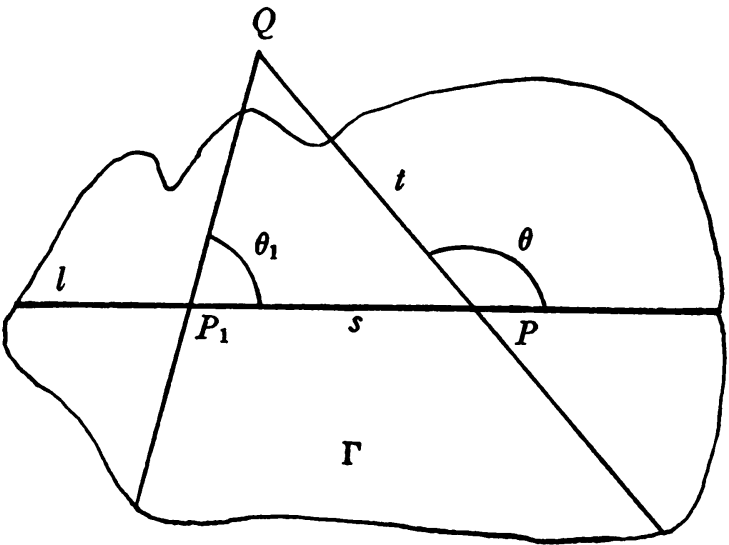

Figure 5

and if $Q$ does not exist, we have

$$
\sin \left(\theta-\theta_{1}\right)=0
$$

As $P \rightarrow P_{1}, t$ is always greater than a positive constant $a$, since $Q$ is not in $\Gamma$ and $P$ is in some closed neighborhood of $P_{1}$. Thus, by (1) and (2), as $P \rightarrow P_{1}$, $\sin \left(\theta-\theta_{1}\right) \rightarrow 0$. We have either

$$
\left|\theta-\theta_{1}\right|<\pi-\theta_{1}
$$

or

$$
\left|\theta-\theta_{1}\right|<\theta_{1}
$$

since $0<\theta<\pi$ and $0<\theta_{1}<\pi$. Thus

$$
\left|\theta-\theta_{1}\right|<b<\pi
$$

so that as $P \rightarrow P_{1}$,

$$
\left(\theta-\theta_{1}\right) \rightarrow 0 \text { and } \theta \rightarrow \theta_{1}
$$

LEMMA IV. If $f^{\prime}$ and $f$ are two lines of a family $f$, and $l, m$ are transversals to $f^{\prime}$ at the same point, the ratio of the distances intercepted on $l$ and $m$ by $f$ and $f^{\prime}$ converges to a non-zero limit as $f \rightarrow f^{\prime}$.*

* That is, the ratio approaches the same limit for all sequences $\left\{f_{n}\right\}$ which converge on $f^{\prime}$. 
Let $P$ (Figure 6) be the common intersection of $l$ and $m$ with $f^{\prime}$, and $L$, $M$ be the respective intersections of $l, m$ with $f$. If $L=M$, the theorem is trivial. If $L \neq M$, we have

$$
P L / P M=\sin \beta / \sin \alpha,
$$

where $\alpha$ and $\beta$ are the inclinations of $f$ with regard to $l$ and $m$ respectively. As $f \rightarrow f^{\prime}, M \rightarrow P$ and $L \rightarrow P$ by Lemma II, so that by Lemma III, $\alpha$ and $\beta$ converge to limits neither of which is zero or $\pi$, since both $l$ and $m$ are distinct from $f^{\prime}$. Thus $P L / P M$ approaches a non-zero limit as $f \rightarrow f^{\prime}$.

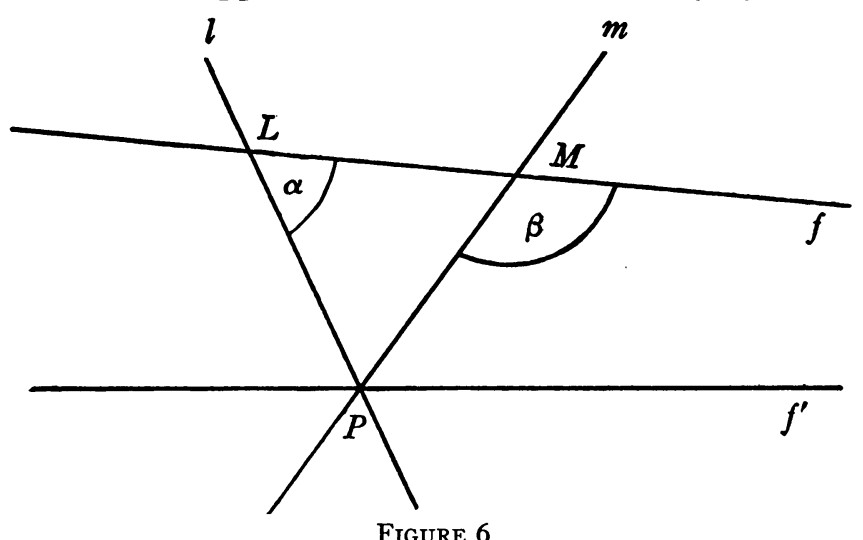

LEMMA V. If $f^{\prime}$ and $f$ are two lines of a family $f$ and $l$, $m$ are transversals to $f^{\prime}$, the ratio of the distances intercepted on $l$ and $m$ by $f$ and $f^{\prime}$ has an upper bound and a positive lower bound as $f \rightarrow f^{\prime}$.*

Let family $f$ be contained in region $\Gamma$ and let $l, m$ intersect $f$ in $L, M$ and $f^{\prime}$ in $L^{\prime}, M^{\prime}$ respectively. If $L^{\prime}=M^{\prime}$, the result is immediate, by Lemma IV.

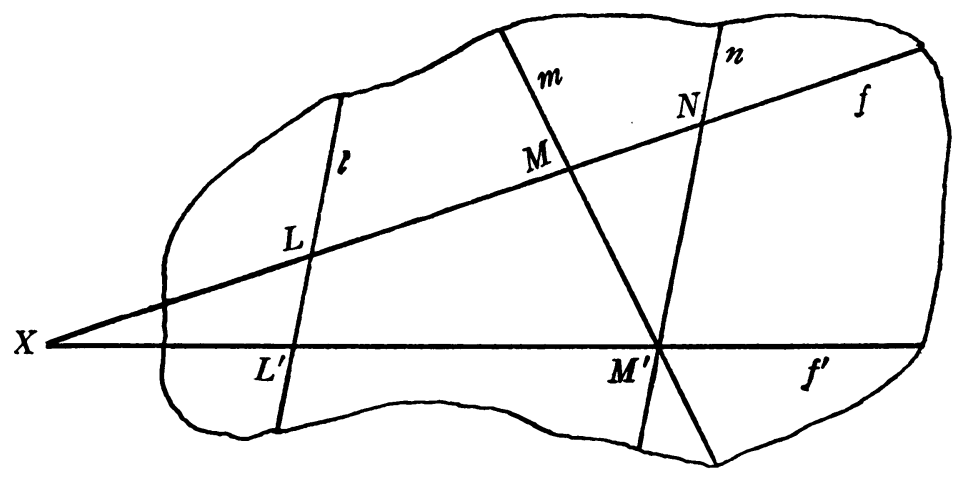

FIGURE 7

* That is, the ratio has fixed bounds for all sequences $\left\{f_{n}\right\}$ which converge on $f^{\prime}$. 
If $L^{\prime} \neq M^{\prime}$ (see Figure 7), consider the line $n$ through $M^{\prime}$ parallel to $l$. Since $n$ intersects $f^{\prime}$, it intersects all $f$ sufficiently close to $f^{\prime}$ by Lemma I. Let $N$ be the intersection of $n$ with $f$. Then, we have

$$
L^{\prime} L / M^{\prime} M=\left(L^{\prime} L / M^{\prime} N\right)\left(M^{\prime} N / M^{\prime} M\right),
$$

and $M^{\prime} N / M^{\prime} M$ converges as $f \rightarrow f^{\prime}$, by Lemma IV. Consider $L^{\prime} L / M^{\prime} N$. Let the intersection of the infinite lines $L N$ and $L^{\prime} M^{\prime}$ be $X$, if it exists. Then, if $X$ exists,

$$
L^{\prime} L / M^{\prime} N=X L^{\prime} / X M^{\prime}=\left(X M^{\prime} \pm L^{\prime} M^{\prime}\right) / X M^{\prime}=1 \pm\left(L^{\prime} M^{\prime} / X M^{\prime}\right),
$$

and if $X$ does not exist,

$$
L^{\prime} L / M^{\prime} N=1 .
$$

But $L^{\prime} M^{\prime}$ is fixed and $X M^{\prime}>a>0$ since $X$ is not in $\Gamma$. Thus $L^{\prime} L / M^{\prime} N$ is bounded above and the same is true of $L^{\prime} L / M^{\prime} M$. By the identical argument $M^{\prime} M / L^{\prime} L$ is bounded above, so that $L^{\prime} L / M^{\prime} M$ has a positive lower bound.

Definition. Let $P$ be a point of the region of definition of a uniform transformation $T, l$ a line containing $P$, and $Q$ a point on $l$. Let $P^{\prime}, Q^{\prime}$ be the images under $T$ of $P, Q$ respectively. Then if $\lim _{Q \rightarrow P} P^{\prime} Q^{\prime} / P Q$ exists, it is called the directional derivative (abbreviated D.D.) of $T$ at $P$ in the direction l.* The D.D. of $T$ at $P$ in the direction $f_{P}$ is called the D.D. of $T$ at $P$ in the direction of family $f$.

LEMMA VI. If a topological transformation T carries $w, a$ 3-web of lines, into a 3-web of lines, then

(a) the D.D. of $T$ exists at almost all points of $l$, any line of $w, \dagger$ in the direction $l$;

(b) if the D.D. of $T$ exists at a point in the direction of one family of $w$, it exists at that point in the direction of each family of w;

(c) the D.D. of $T$ is not zero at any point of w

(a) Since the transformation is topological, betweenness is preserved for points on $l$, any line of $w$. Thus, if we establish scales of ordinates on $l$ and on its image, the ordinate $x^{\prime}$ of the image point is a monotonic function of $x$, the ordinate of the given point. Therefore, by a theorem of Lebesgue, the derivative of $x^{\prime}$ with regard to $x$ exists and is finite almost everywhere on $l$. Since the absolute value of the derivative $d x^{\prime} / d x$ at a point of $l$ is the D.D. of $T$ at this point in the direction $l$, conclusion (a) is true.

\footnotetext{
* Note that the phrase is defined only if $l$ contains $P$.

$\dagger$ The lines of the families which constitute a web are called lines of the web, and the points of these lines, points of the web.
} 
(b) Let the three families which form $w$ be $f, g$, and $h$. Then it will suffice to show that if the D.D. of $T$ exists at $P$ (Figure 8 ) in the direction $f_{P}$, it also exists at $P$ in the direction $g_{P}$. Let $Q$ be any point on $g_{P}$, close to $P$. Then $h_{Q}$ intersects $f_{P}$ at $R$ by Lemma I. Let the images of $P, Q, R$ be $P^{\prime}, Q^{\prime}, R^{\prime}$ respectively. Then we have

$$
P^{\prime} Q^{\prime} / P Q=\left(P^{\prime} Q^{\prime} / P^{\prime} R^{\prime}\right)\left(P^{\prime} R^{\prime} / P R\right)(P R / P Q) .
$$

The fractions $P^{\prime} Q^{\prime} / P^{\prime} R^{\prime}$ and $P R / P Q$ converge as $Q \rightarrow P$ in view of Lemma IV. As $Q \rightarrow P, R \rightarrow P$ also, by Lemma II. Thus $P^{\prime} R^{\prime} / P R$ converges as $Q \rightarrow P$. Therefore, the desired D.D. which is $\lim _{Q \rightarrow P} P^{\prime} Q^{\prime} / P Q$ exists.

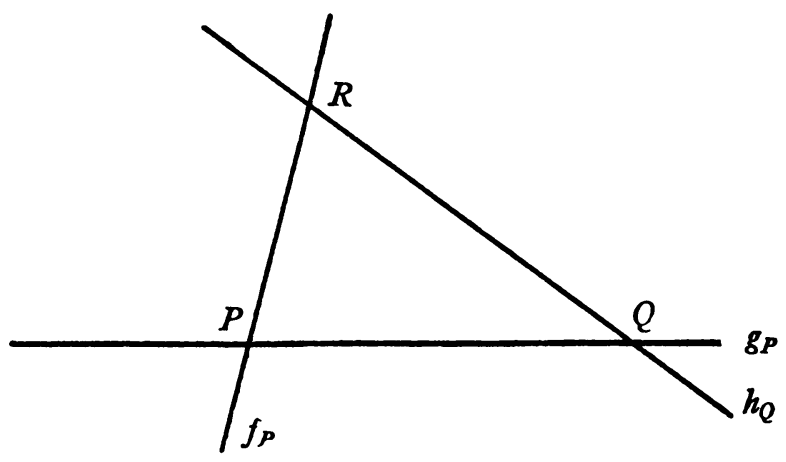

FIGURE 8

(c) Suppose that the D.D. of $T$ is zero at some point in the direction of a line of the web, let us say at point $P$ in the direction $f_{P}$. We notice from (1) in the proof of (b), that if $P^{\prime} R^{\prime} / P R \rightarrow 0, P^{\prime} Q^{\prime} / P Q \rightarrow 0$ likewise. In other words, if the D.D. of $T$ is zero at a point in the direction of one line of the web, it is zero at that point in the direction of each line of the web through that point. We shall use this to show that the D.D. of $T$ is zero in the direction $f_{P}$, at each point of $f_{P}$.

Let $Q$ (Figure 9) be any point on $f_{P}$ distinct from $P$. Then if $R$ is an arbitrary point on $g_{Q}$, sufficiently close to $Q, f_{R}$ intersects $g_{P}$ at $S$. Let the corresponding image points be $P^{\prime}, Q^{\prime}, R^{\prime}$, and $S^{\prime}$. We have

$$
Q^{\prime} R^{\prime} / Q R=\left(Q^{\prime} R^{\prime} / P^{\prime} S^{\prime}\right)\left(P^{\prime} S^{\prime} / P S\right)(P S / Q R) .
$$

The first and third factors of (1) are bounded as $R \rightarrow Q$ in view of Lemma $V$, and the second factor approaches zero. Thus, the D.D. of $T$ at $Q$ in the direction $g_{Q}$ is zero, whence it is also zero at $Q$ in the direction $f_{P}$. But since $Q$ is arbitrary, the D.D. of $T$ is zero everywhere on $f_{P}$ in the direction $f_{P}$, which implies that the image of $f_{P}$ is a single point. Thus our original supposition is false and (c) is proved. 


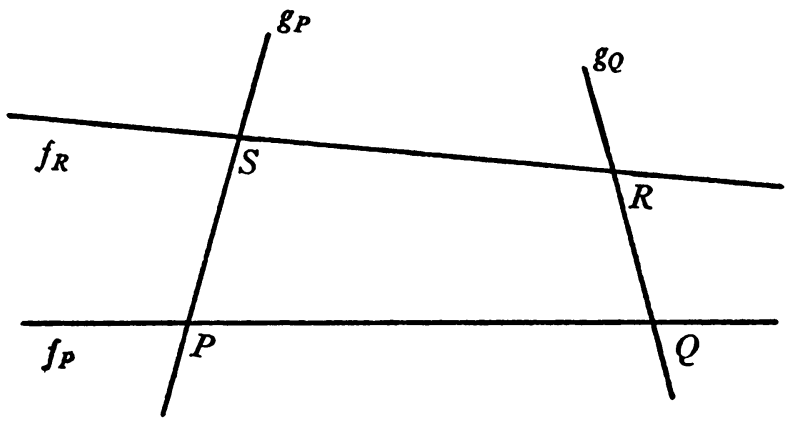

Figure 9

DeFinition. If $f_{1}$ is a line of family $f$, such that the ratio of the distances intercepted by $f$ and $f_{1}$ on each pair of transversals to $f_{1}$ converges as $f \rightarrow f_{1}$, the family $f$ is said to be regular at $f_{1}$. A regular family is one which is regular at each of its lines, and a web is called regular if each of its families is regular.

Regular families are important in this paper mainly because their slope functions are differentiable, as is shown in Lemma XII. An example of a regular family is a set of parallel lines in a circular region; the family consisting of the lines

$$
y=k \quad(1 \leqq k<2), \quad y=l(x-2)+1 \quad(0<l<\infty)
$$

contained in the triangular region whose vertices are $(0,0),(2,0),(2,2)$ is not regular at the line $y=1$.

LEMMA VII. A sufficient condition that family $f$ be regular at $f_{1}$, is that the ratio of the distances intercepted by $f$ and $f_{1}$ on a single pair of transversals which intersect $f_{1}$ at different points, converge as $f \rightarrow f_{1}$.

Let $m, n$ (Figure 10) be the two given transversals, and $r, s$ any two transversals to $f_{1}$, and let their respective intersections with $f_{1}, f$ be $M_{1}, M, N_{1}$, $N, R_{1}, R, S_{1}, S$. We have to derive the existence of $\lim _{f \rightarrow f_{1}} R R_{1} / S S_{1}$ from that of $\lim _{f \rightarrow f_{1}} M M_{1} / N N_{1}$. Draw parallel transversals to $f_{1}$ at $M_{1}, N_{1}, R_{1}, S_{1}$, which intersect $f$ at $\bar{M}, \bar{N}, \bar{R}, \bar{S}$ respectively.

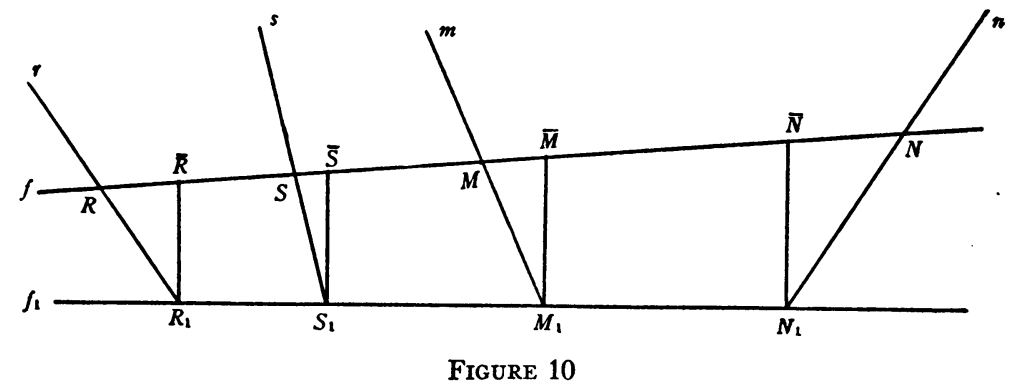


Then we have

$$
\bar{M} M_{1} / \bar{N} N_{1}=\left(\bar{M} M_{1} / M M_{1}\right)\left(M M_{1} / N N_{1}\right)\left(N N_{1} / \bar{N} N_{1}\right) .
$$

The first and third factors on the right in (1) converge as $f \rightarrow f_{1}$, by Lemma IV; thus $\bar{M} M_{1} / \bar{N} N_{1}$ converges as $f \rightarrow f_{1}$. In the same way, we show that the existence of $\lim _{f \rightarrow f_{1}} \bar{R} R_{1} / \bar{S} S_{1}$ implies that of $\lim _{f \rightarrow f_{1}} R R_{1} / S S_{1}$. Thus we have only to prove the existence of $\lim _{f \rightarrow f_{1}} \bar{R} R_{1} / \bar{S} S_{1}$.

By the point of division formula of elementary analytic geometry, we have

$$
\begin{array}{r}
\frac{\bar{R} R_{1}}{\bar{S} S_{1}}=\frac{\bar{M} M_{1}+a \bar{N} N_{1}}{1+a} \div \frac{\bar{M} M_{1}+b \bar{N} N_{1}}{1+b}=\frac{\bar{M} M_{1} / \bar{N} N_{1}+a}{\bar{M} M_{1} / \bar{N} N_{1}+b} \cdot \frac{1+b}{1+a} \\
(a \neq-1, b \neq-1) .
\end{array}
$$

In addition

$$
\left|\left(\bar{M} M_{1} / \bar{N} N_{1}\right)+b\right|=\left|\left(\bar{S} S_{1} / \bar{N} N_{1}\right)(1+b)\right|>c>0
$$

where $c$ is constant, by Lemma V. Thus, by (2) the convergence of $\bar{M} M_{1} / \bar{N} N_{1}$ as $f \rightarrow f_{1}$ implies that of $\bar{R} R_{1} / \bar{S} S_{1}$ and the lemma is true.

LEMMA VIII. If a topological transformation carries a regular 3-web of lines into a 3-web of lines, the latter is regular also.*

Let $f$ and $g$ be any two families of the given web, and $f^{\prime}$ the image of $f$. It will be sufficient to show that $f^{\prime}$ is regular at $f_{1}^{\prime}$, any one of its lines. Let $f_{1}^{\prime}$ be the image of $f_{1}$. Choose distinct points $P, Q$ on $f_{1}$, at each of which the D. D. of the transformation exists in the direction $f_{1}$. Let $g_{P}, g_{Q}$ intersect $f$, an arbitrary line of family $f$, at $R, S$ respectively. Let $f^{\prime}$ be the image of line $f$, and $P^{\prime}, Q^{\prime}, R^{\prime}, S^{\prime}$, the images of $P, Q, R, S$, respectively.

Then

$$
\frac{P^{\prime} R^{\prime}}{Q^{\prime} S^{\prime}}=\left(\frac{P^{\prime} R^{\prime}}{P R} \cdot \frac{P R}{Q S}\right) \div \frac{Q^{\prime} S^{\prime}}{Q S} .
$$

We know that $\lim _{R \rightarrow P} P^{\prime} R^{\prime} / P R$ and $\lim _{S \rightarrow Q} Q^{\prime} S^{\prime} / Q S$ exist and the latter is not zero, by Lemma VI (b), (c), and $\lim _{f \rightarrow f_{1}} P R / Q S$ exists, by hypothesis. However, $f^{\prime} \rightarrow f_{1}^{\prime}$ implies that $f \rightarrow f_{1}$ and hence that $R \rightarrow P$ and $S \rightarrow Q$ by Lemma II. Therefore $\lim _{f^{\prime} \rightarrow f_{1}} P^{\prime} R^{\prime} / Q^{\prime} S^{\prime}$ exists and the result follows by Lemma VII.

LEMMA IX. If a topological transformation carries w, a regular 3-web of lines, into a 3-web of lines, the D.D. of the transformation exists at each point of $w$, in the directions of the lines of $w$.

* The proof which follows also justifies the more general result: If a topological transformation carries w, a 3-web of lines, ints a 3-web of lines, and a family of $w$ is regular at a line, then the image of the family is regular at the image of the line. 
Let $f$ and $g$ be any two families of $w$. We shall show that the D.D. exists at $P$, any point of $w$, in the direction of $f$. There is a point $Q$, distinct from $P$, on $f_{P}$ at which the D.D. exists in the direction of $f$. Choose an arbitrary point $R$ on $g_{P}$, distinct from $P$ and close enough to $P$ so that $f_{R}$ intersects $g_{Q}$ at $S$. Denoting image points in the usual way, we have

$$
\left(P^{\prime} R^{\prime} / P R\right)=\left(P^{\prime} R^{\prime} / Q^{\prime} S^{\prime}\right)\left(Q^{\prime} S^{\prime} / Q S\right)(Q S / P R) .
$$

Now we can show that the three factors on the right converge as $R \rightarrow P$, the first by means of Lemma VIII, the second by Lemma VI(b), and the third directly by the hypothesis. Thus $\lim _{R \rightarrow P} P^{\prime} R^{\prime} / P R$, i.e., the D.D. of the transformation at $P$ in the direction of $g$, exists, which implies its existence at $P$ in the direction of $f$, and the lemma is proved.

LEMMA X. If a topological transformation of region $\Gamma$ carries a regular 3-web of lines in $\Gamma$ into a 3-web of lines, it is differentiable and its Jacobian is nowhere zero.

We shall show that if there are established arbitrary rectangular coordinate systems in the given and image planes, the functions representing the transformation are differentiable and have a nowhere vanishing Jacobian. However, if we prove that the transformation is differentiable and its Jacobian is not zero at $P$, an arbitrary point of $\Gamma$, for special cartesian axes dependent on $P$, the above result will follow. For the change of coordinates which must be applied to shift from the special axes to those originally chosen is a non-singular linear transformation, and hence is differentiable with a nonvanishing Jacobian.

Let $f, g$ be two families of the given web and let their images be $u, v$ respectively. Take $f_{P}, g_{P}$ (Figure 11) as the special cartesian axes and set up a coordinate system using an arbitrary unit distance and arbitrary positive directions on $f_{P}$ and $g_{P}$. We represent image points as heretofore. In the image plane, we establish a similar coordinate system using $u_{P^{\prime}}$ and $v_{P^{\prime}}$ as coordinate axes and choosing an arbitrary unit distance. However, the positive directions on $u_{P^{\prime}}$ and $v_{P^{\prime}}$ are taken to be the images of the positive directions on $f_{P}$ and $g_{P}$ respectively.

Let $Q(x, y)$ be any point in the neighborhood of $P$ and $Q^{\prime}\left(x^{\prime}, y^{\prime}\right)$ its image. We shall show that $x^{\prime}$ and $y^{\prime}$ are differentiable functions of $x$ and $y$, at $(0,0)$. Let $g_{Q}$ intersect $f_{P}$ in $R$, and $v_{Q^{\prime}}$ intersect $u_{P^{\prime}}$ in $R^{\prime}$.

If $R$ is in the positive (negative) direction on $f_{P}$ from $P$, then $R^{\prime}$ is in the positive (negative) direction on $u_{P^{\prime}}$ from $P^{\prime}$. Moreover, if $R$ is in the positive (negative) direction from $P, x$ is positive (negative) and a similar relation holds for $R^{\prime}$ and $x^{\prime}$. Thus, $x$ and $x^{\prime}$ have the same sign. 


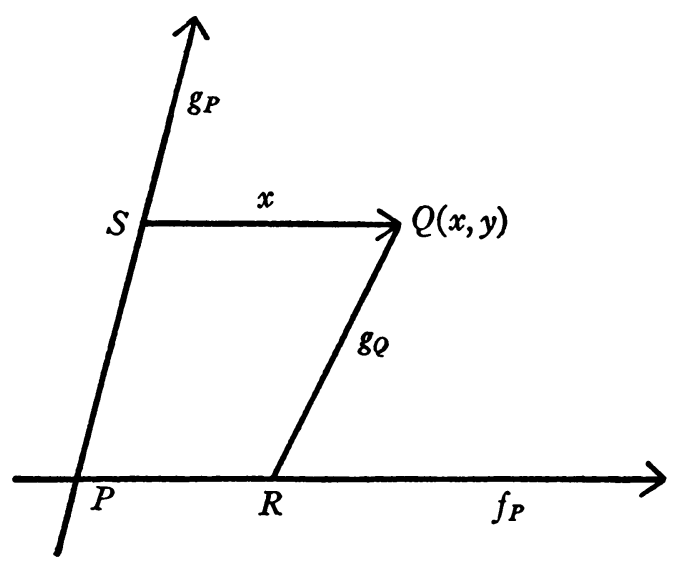

FigURE 11

Now suppose that $Q$ is not on $g_{P}$, so that $x \neq 0$. Then we have

$$
x^{\prime}=\left(\left|x^{\prime}\right| / P^{\prime} R^{\prime}\right)\left(P^{\prime} R^{\prime} / P R\right)(P R /|x|) x .
$$

As $Q \rightarrow P, R \rightarrow P$ and $P^{\prime} R^{\prime} / P R \rightarrow a$, the D.D. of the transformation at $P$ in the direction of $f$. Also, as $Q \rightarrow P$, the first and third factors of the right member of (1) converge to unity. We shall prove this for $P R /|x|$; the proof is similar for $\left|x^{\prime}\right| / P^{\prime} R^{\prime}$.

Let $S$ be the intersection of $g_{P}$ and the line through $Q$ parallel to $f_{P}$. Then either

or

$$
P R=|x|
$$

$$
P R /|x|=P T / S T=(S T \pm P S) / S T=1 \pm(P S / S T),
$$

where $T$ is the intersection of the extensions of $g_{P}$ and $g_{Q}$. As $Q \rightarrow P, P S \rightarrow 0$ and $S T>k>0$, so that $P R /|x| \rightarrow 1$.

Therefore for $Q$ not on $g_{P}$, we have

$$
x^{\prime}=\left(1+\epsilon_{1}\right)\left(a+\epsilon_{2}\right)\left(1+\epsilon_{3}\right) x=a x+\epsilon x,
$$

where $a \neq 0$ by Lemma VI (c) and $\epsilon \rightarrow 0$ as $Q \rightarrow P$. If $Q$ is on $g_{P}, x=x^{\prime}=0$, so that (2) holds in this case with $\epsilon=0$. Therefore (2) holds for all $(x, y)$ in the neighborhood of $(0,0)$, and $\epsilon$ approaches zero with $x$ and $y$. Similarly

$$
y^{\prime}=b y+\eta y
$$

where $b$ is the D.D. of the transformation at $P$ in the direction $g_{P}$ and hence is not zero, and $\eta$ vanishes with $x$ and $y$. Thus by definition $x^{\prime}$ and $y^{\prime}$ are 
differentiable functions of $x$ and $y$ at $(0,0)$. Moreover, the Jacobian at $(0,0)$ is

$$
\left|\begin{array}{ll}
a & 0 \\
0 & b
\end{array}\right| \neq 0,
$$

and the desired result follows.

LEMмa XI. If a topological transformation $T$ carries w, a 3-web of lines, into a 3-web of lines, and a family of $w$ is not regular at one of its lines, then $T$ is a projectivity on this line.

Let two families of the given web be $f$ and $g$ and suppose that $f$ is not regular at $f_{1}$. Let $P_{1}, P_{2}, P$ (Figure 12) be distinct points on $f_{1}$ at which the D.D. of $T$ in the direction $f_{1}$ exists; $P_{1}$ and $P_{2}$ are to be fixed and $P$ is a variable point. Consider three parallel transversals to $f_{1}$ at $P_{1}, P_{2}, P$ respectively, and let their intersections with $f$, a variable line of $f$ distinct from $f_{1}$, be $R_{\mathbf{1}}, R_{\mathbf{2}}$, $R$ respectively. Let $f_{1}^{\prime}, f^{\prime}, P_{1}^{\prime}, P_{2}^{\prime}, P^{\prime}$ be the images of $f_{1}, f, P_{1}, P_{2}, P$ respectively. Consider three parallel transversals to $f_{1}^{\prime}$ at $P_{1}^{\prime}, P_{2}^{\prime}, P^{\prime}$ meeting $f^{\prime}$ in $\bar{R}_{1}, \bar{R}_{2}, \bar{R}$. Let $s_{1}, s_{2}, s, \bar{s}_{1}, \bar{s}_{2}, \bar{s}$ represent the respective distances $P_{1} R_{1}, P_{2} R_{2}$, $P R, P_{1}^{\prime} \bar{R}_{1}, P_{2}^{\prime} \bar{R}_{2}, P^{\prime} \bar{R}$.

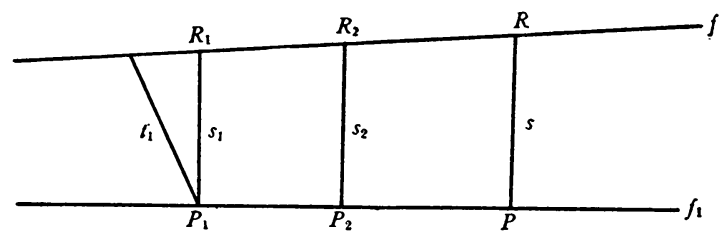

FigURe 12

Then, as $f \rightarrow f_{1}$, the ratios $\bar{s}_{1} / s_{1}, \bar{s}_{2} / s_{2}, \bar{s} / s$ approach non-zero limits. We shall show this for $\bar{s}_{1} / s_{1}$. Let $t_{1}$ be the distance intercepted on $g_{P}$ by $f_{1}$ and $f$, and $t_{1}^{\prime}$ the image distance. Then $\lim _{f \rightarrow f_{1}} t_{1}^{\prime} / t_{1}$ exists and is not zero by Lemma VI (b), (c), since the D.D. of $T$ exists at $P_{1}$ in the direction $f_{1}$. By Lemma IV, as $f \rightarrow f_{1}, \bar{s}_{1} / t_{1}^{\prime}$ and $t_{1} / s_{1}$ converge to values different from zero. Thus, since

$$
\bar{s}_{1} / s_{1}=\left(\bar{s}_{1} / t_{1}^{\prime}\right)\left(t_{1}^{\prime} / t_{1}\right)\left(t_{1} / s_{1}\right),
$$

$\bar{s}_{1} / s_{1}$ converges to a non-zero value as $f \rightarrow f_{1}$.

We have

$$
\begin{array}{ll}
s=\left(s_{1}+\lambda s_{2}\right) /(1+\lambda) & (\lambda \neq-1), \\
\bar{s}=\left(\bar{s}_{1}+\lambda^{\prime} \bar{s}_{2}\right) /\left(1+\lambda^{\prime}\right) & \left(\lambda^{\prime} \neq-1\right),
\end{array}
$$

where $\lambda, \lambda^{\prime}$ are the ratios in which $P, P^{\prime}$ divide the directed segments $P_{1} P_{2}$, $P_{1}^{\prime} P_{2}^{\prime}$ respectively. By division, we obtain 


$$
\frac{\bar{s}}{s}=b \frac{\bar{s}_{1}+\lambda^{\prime} \bar{s}_{2}}{s_{1}+\lambda s_{2}}
$$

where

$$
b=(1+\lambda) /\left(1+\lambda^{\prime}\right) \neq 0 .
$$

Then if $a_{1}, a_{2}$ are the respective limits of $\bar{s}_{1} / s_{1}, \bar{s}_{2} / s_{2}$ as $f \rightarrow f_{1}$,

$$
\bar{s}_{1}=a_{1} s_{1}+\epsilon_{1} s_{1} \quad\left(a_{1} \neq 0\right)
$$

and

$$
\bar{s}_{2}=a_{2} s_{2}+\epsilon_{2} s_{2} \quad\left(a_{2} \neq 0\right),
$$

where $\epsilon_{1} \rightarrow 0$ with $s_{1}$ and $\epsilon_{2} \rightarrow 0$ with $s_{2}$. Thus, substituting in (1), we have

and

$$
\frac{\bar{s}}{s}=b \frac{a_{1} s_{1}+\epsilon_{1} s_{1}+\lambda^{\prime} a_{2} s_{2}+\lambda^{\prime} \epsilon_{2} s_{2}}{s_{1}+\lambda s_{2}}
$$

$$
\frac{\bar{s}}{s}=b a_{1}+b \frac{\epsilon_{1} s_{1}+\lambda^{\prime} \epsilon_{2} s_{2}}{s_{1}+\lambda s_{2}}+b \frac{\left(\lambda^{\prime} a_{2}-\lambda a_{1}\right) s_{2}}{s_{1}+\lambda s_{2}} .
$$

The second term of the right member of (2) converges as $f \rightarrow f_{1}$. For, if we divide its numerator and denominator by $s_{2}$, we have

$$
b \frac{\epsilon_{1}\left(s_{1} / s_{2}\right)+\lambda^{\prime} \epsilon_{2}}{\left(s_{1} / s_{2}\right)+\lambda}
$$

the numerator of which approaches zero as $f \rightarrow f_{1}$, since, by Lemma $\mathrm{V}, s_{1} / s_{2}$ is bounded; and the absolute value of the denominator

$$
\left|\left(s_{1} / s_{2}\right)+\lambda\right|=\left|\left(s_{1}+\lambda s_{2}\right) / s_{2}\right|=\left|c s / s_{2}\right|>d>0
$$

where $c$ and $d$ are constants, by Lemma V. Thus, the third term on the right in (2) converges as $f \rightarrow f_{1}$. But this term is

$$
b \frac{\left(\lambda^{\prime} a_{2}-\lambda a_{1}\right) s_{2}}{s_{1}+\lambda s_{2}}=b \frac{\lambda^{\prime} a_{2}-\lambda a_{1}}{\left(s_{1} / s_{2}\right)+\lambda},
$$

and $s_{1} / s_{2}$ does not converge as $f \rightarrow f_{1}$, since family $f$ is not regular at $f_{1}$. Thus

$$
\lambda^{\prime} a_{2}-\lambda a_{1}=0
$$

and

$$
\lambda^{\prime}=\left(a_{1} / a_{2}\right) \lambda \text {. }
$$

Now we may consider $\lambda, \lambda^{\prime}$ to be the projective ordinates of $P, P^{\prime}$ on 
the lines $f_{1}, f_{1}^{\prime}$ respectively. Thus by (3), the transformation is a projectivity on $f_{1}$ for the everywhere dense set of points $P$, and hence for all points.

DEFINITION. If a family of lines $f$ is referred to rectangular coordinate axes, the slope of $f_{P}$, considered as a function of the coordinates of $P$, is called the slope function of the family $f$. The slope function is not defined at any point of a vertical line of the family.

LeMma XII. The slope function of a regular family of lines is differentiable.*

Let the family be $f$, contained in $\Gamma$, and let $P\left(x_{1}, y_{1}\right)$ be any point of $\Gamma$ at which $m(x, y)$, the slope function of $f$, exists. We shall show that $m(x, y)$ is differentiable at $\left(x_{1}, y_{1}\right)$. Let $Q(x, y)$ (Figure 13) be any point in the neighborhood of $P$. Then the line $x=x_{1}$ intersects $f_{Q}$ at $\left(x_{1}, y_{1}+d_{1}\right)$. If $x_{2}$ is distinct from but close to $x_{1}$, the line $x=x_{2}$ intersects $f_{P}$ and $f_{Q}$ at $\left(x_{2}, y_{2}\right)$ and $\left(x_{2}, y_{2}+d_{2}\right)$, respectively. We note that $d_{1}$ and $d_{2}$ have the same algebraic sign.

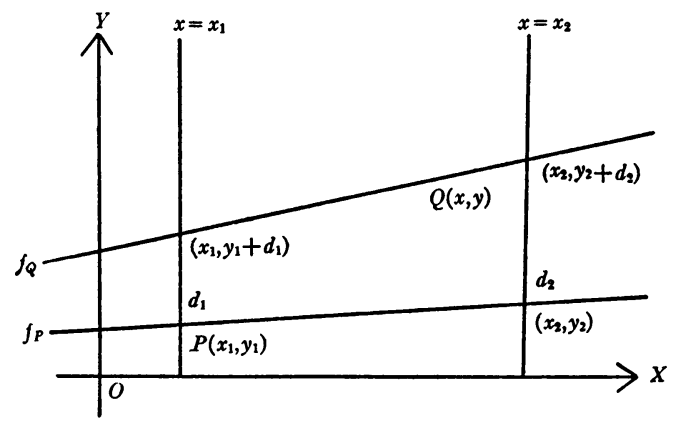

FIGURE 13

By Lemma I, $f_{Q}$ intersects $x=x_{1}$, and hence has a slope, $m(x, y)$. We write $m_{1}, m$ for $m\left(x_{1}, y_{1}\right), m(x, y)$ respectively. Suppose that $Q$ is not on $f_{P}$. We may assert that

$$
m=\frac{y_{2}+d_{2}-\left(y_{1}+d_{1}\right)}{x_{2}-x_{1}}
$$

and

$$
m_{1}=\frac{y_{2}-y_{1}}{x_{2}-x_{1}} .
$$

Hence, we have

$$
m-m_{1}=a\left(d_{2}-d_{1}\right)
$$

* The proof which follows also justifies the more general result: The slope function of family $f$ is differentiable at $P\left(x_{1}, y_{1}\right)$, if $f$ is regular at $f_{P}$. 
and

$$
m=m_{1}+a\left(d_{2}-d_{1}\right),
$$

where $a=1 /\left(x_{2}-x_{1}\right)$. We may easily get

$$
d_{1}=\Delta y-m \Delta x
$$

where $\Delta y=y-y_{1}$ and $\Delta x=x-x_{1}$. Substituting for $m$ in (3) its value in (2), we have

$$
d_{1}=\Delta y-m_{1} \Delta x-a\left(d_{2}-d_{1}\right) \Delta x .
$$

Since $d_{1} \neq 0$, we may obtain from (1) and (4)

$$
m-m_{1}=\frac{a\left(d_{2}-d_{1}\right)}{d_{1}}\left[\Delta y-m_{1} \Delta x-a\left(d_{2}-d_{1}\right) \Delta x\right] .
$$

Since family $f$ is regular, $\lim _{Q \rightarrow P} d_{2} / d_{1}$ exists and hence $\lim _{Q \rightarrow P}\left(d_{2}-d_{1}\right) / d_{1}$ exists. Therefore

$$
m-m_{1}=\left(b+\epsilon_{1}\right)\left(\Delta y-m_{1} \Delta x+\epsilon_{2} \Delta x\right),
$$

and we have

$$
m-m_{1}=b\left(\Delta y-m_{1} \Delta x\right)+\epsilon \Delta x+\eta \Delta y,
$$

where $b$ is constant and $\epsilon$ and $\eta$ vanish with $\Delta x$ and $\Delta y$.

If $Q$ is on $f_{P}$, we have

$$
m-m_{1}=b\left(\Delta y-m_{1} \Delta x\right)=0 .
$$

Consequently (5) holds for all $Q$, and $\epsilon$ and $\eta$ vanish with $\Delta x$ and $\Delta y$ regardless of how the latter approach zero. Thus, by definition, $m(x, y)$ is differentiable at $\left(x_{1}, y_{1}\right)$.

\section{Collineations of REgions OF THE EUClideAN PLANE}

We shall first establish the following result, which is used as a lemma to the succeeding theorems.

THEOREM IV. Any one-to-one transformation of region $\Gamma$ which carries a 3-web of lines in $\Gamma$ into a 3-web of lines is projective, if it is projective in the neighborhood of one point.

Let the given web $w$ consist of families $f, g$, and $h$. Let $P$ be a point of $\Gamma$ in a neighborhood of which the given transformation $T$ is projective. Choose four points, no three of which are collinear in this neighborhood of $P$, and let $S$ be the projective transformation which carries the images of these four points under $T$ into the original points, respectively. Transformation $S$ is de- 
fined for all points of the image web, with the possible exception of points of one line, the so-called vanishing line of the projective transformation.

Let $R$ be the resultant of $T$ and $S$ in that order. Transformation $R$ is defined for all points of $\Gamma$, with the exception of those whose images under $T$ are on the vanishing line of $S$, if such exist; and $R$ carries points on any line of $w$ into collinear points. Moreover, if $R$ is not defined for a point of $\Gamma$, the lines of $w$ which contain this point are carried into sub-sets of parallel lines by $R$. Hence, if $R$ carries any two of $f_{M}, g_{M}, h_{M}$ into sub-sets of lines which intersect at $N, R$ is defined for point $M$ and carries $M$ into $N$.

It is evident that $R$ is the identical transformation in the neighborhood of $P$. We shall obtain the result by showing that $R$ is the identity over the whole region $\Gamma$.

Consider points $X$, contained in $\Gamma$, such that all points in the neighborhood of $X$ are invariant under $R$, and $X$ can be joined to $P$ by a broken line, each point of which has a neighborhood composed of points invariant under $R$. Let $\Gamma_{1}$ be the set consisting of $P$ and all points $X$. It can easily be shown that $\Gamma_{1}$ is a sub-region of $\Gamma$. We shall show that $\Gamma_{1}$ is identical with $\Gamma$, thus proving that $R$ leaves each point of $\Gamma$ invariant.

First we show that if $A$ is a point of $\Gamma$ on the boundary of $\Gamma_{1}$, not more than one of the lines $f_{A}, g_{A}, h_{A}$ contains a point of $\Gamma_{1}$. We shall call this assertion (1). Let us suppose that this is not so. Then there is no loss in generality in assuming that $f_{A}$ and $g_{A}$ each contains a point of $\Gamma_{1}$. It follows that each contains several points of $\Gamma_{1}$. Therefore $R$ carries $f_{A}$ and $g_{A}$ into sub-sets of the respective infinite lines which contain $f_{A}$ and $g_{A}$. This implies that $A$ is an invariant point of $R$.

If $Y$ is any point in a sufficiently small neighborhood of $A, f_{Y}$ and $g_{Y}$ each contains a point of $\Gamma_{1}$, by the corollary to Lemma I. Thus the invariance of $Y$ follows by the argument just used to prove the invariance of $A$. In other words, we have shown that $A$ has a neighborhood consisting of points invariant under $R$. It follows that $A$ can be joined to $P$ by a broken line, with the property that each of its points has a neighborhood composed of invariant points. Because if $Q$, distinct from $P$, is a point of $\Gamma_{1}$ in the neighborhood of $A$, $A$ can be joined to $Q$, and $Q$ to $P$ by broken lines which have the same property. Thus, by definition, $A$ belongs to $\Gamma_{1}$, which is impossible because $A$ is a boundary point of $\Gamma_{1}$. This contradiction establishes our original assertion concerning $A$.

Now, in order to prove that $\Gamma_{1}$ and $\Gamma$ are identical, let us suppose that they are distinct. Then we can show there is a line of the web, which contains a point of $\Gamma_{1}$ and a point of its boundary. For, since $\Gamma_{1}$ is a proper part of $\Gamma$, there is, in $\Gamma$, a point $B$, of the boundary of $\Gamma_{1}$. Then, if $f_{B}$ contains a point 
of $\Gamma_{1}$, it is the desired line. If $f_{B}$ contains no point of $\Gamma_{1}$, we can find $C$, a point of $\Gamma_{1}$, so close to $B$, that $g_{C}$ intersects $f_{B}$ at a point which we call $D$. Since $g_{C}$ contains $C$, a point of $\Gamma_{1}$, and $D$, a point not of $\Gamma_{1}$, it must contain a boundary point of $\Gamma_{1}$. Hence in this case, $g_{C}$ is the desired line.

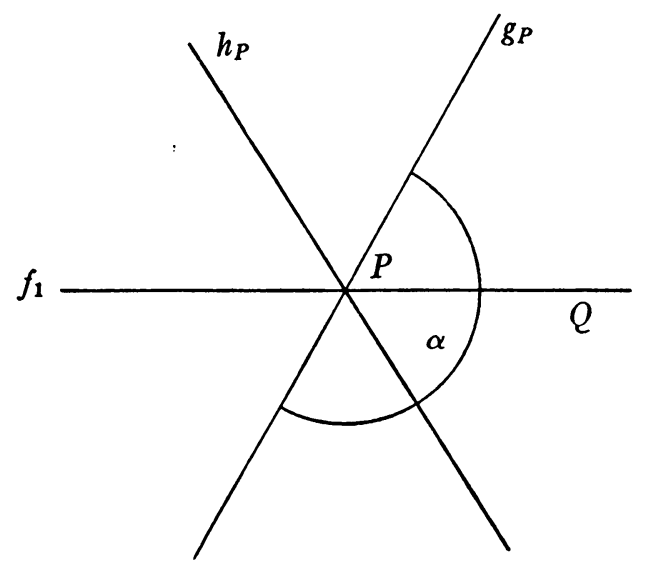

FIGURE 14

Let $f_{1}$ (Figure 14) be a line which contains a point of $\Gamma_{1}$ and a point of its boundary.* Then $f_{1}$ contains point $P$, on the boundary of $\Gamma_{1}$, and point $Q$, such that the segment $P Q$ consists of points of $\Gamma_{1}$. Let $\alpha$ be a semicircular region, with $P$ as center, with diameter on $g_{P}$, and containing a point of segment $P Q$. If each such region $\alpha$ contains a boundary point of $\Gamma_{1}$, we can find $E$, a boundary point of $\Gamma_{1}$, in a region $\alpha$ which is so small that $g_{E}$ intersects $f_{P}$ at a point of segment $P Q$, and $f_{E}$ contains a point of $\Gamma_{1}$. Thus $f_{E}$ and $g_{E}$ each contains a point of $\Gamma_{1}$, which contradicts assertion (1) proved above.

Therefore, there exists a semicircular region $\alpha$, which contains no boundary point of $\Gamma_{1}$. Since this region contains one point of $\Gamma_{1}$, it can contain only points of $\Gamma_{1}$. Hence $h_{P}$, which contains a point of each region $\alpha$, contains a point of $\Gamma_{1}$. But this also is inconsistent with assertion (1), since $f_{P}$ contains a point of $\Gamma_{1}$, and $P$ is on the boundary of $\Gamma_{1}$.

Hence, the assumption that $\Gamma$ and $\Gamma_{1}$ are distinct is false, and the truth of the theorem follows.

Now we shall prove the principal theorem.

THEOREM V. Any topological transformation of region $\Gamma$ which carries a 4-web of lines in $\Gamma$ into a 4-web of lines is a projective collineation.

* There is no loss of generality in supposing that family $f$ contains a line of this type. 
We need merely show, in view of Theorem IV, that the transformation is a projective collineation in the neighborhood of one point of $\Gamma$.

First, we shall prove the theorem on the supposition that $\Gamma$ contains a sub-region $\Gamma_{1}$, in which three families of the given web are regular. ${ }^{*}$ Let $P$ be any point of $\Gamma_{1}$. Choose rectangular coordinate axes in the given and image planes so that the lines of the web through $P$ and their image lines have slopes. Then, in view of Lemma $I, \Gamma_{1}$ contains $V$, a circular neighborhood of $P$, such that the lines of the given and image webs have slopes, if they contain points of $V$ or of $V^{\prime}$, its image.

By Lemma $\mathrm{X}$, we can extend the transformation in $V$, and get

$$
\frac{d y^{\prime}}{d x^{\prime}}=\frac{p_{1}+p_{2}(d y / d x)}{p_{3}+p_{4}(d y / d x)}
$$

where the $p$ 's are the partial derivatives of $x^{\prime}, y^{\prime}$, the coordinates of the image of $(x, y)$, and the Jacobian

$$
J=\left|\begin{array}{ll}
p_{1} & p_{2} \\
p_{3} & p_{4}
\end{array}\right|
$$

does not vanish in $V$. Let $m_{i}(x, y)$ be the slope functions of the three given regular families in $V$, and $m_{i}^{\prime}\left(x^{\prime}, y^{\prime}\right)$ the slope functions in $V^{\prime}$ of their respective image families, which also are regular by Lemma VIII. These six slope functions are differentiable by Lemma XII. Moreover, $m_{i}^{\prime}$ is differentiable with respect to $x$ and $y$, since it is differentiable with respect to $x^{\prime}$ and $y^{\prime}$ which are differentiable with respect to $x$ and $y$.

Then

$$
m_{i}^{\prime}\left(x^{\prime}, y^{\prime}\right)=\frac{p_{1}+p_{2} m_{i}(x, y)}{p_{3}+p_{4} m_{i}(x, y)} \quad(i=1,2,3),
$$

the denominator of which cannot vanish since $m_{i}^{\prime} \neq \infty$ and $J$ does not vanish. Thus, we have

$$
p_{1}+p_{2} m_{i}-p_{3} m_{i}{ }^{\prime}-p_{4} m_{i} m_{i}{ }^{\prime}=0 \quad(i=1,2,3),
$$

which we shall solve for the $p$ 's.

The matrix of the system (2),

$$
\left\|\begin{array}{cccc}
1 & m_{1} & -m_{1}^{\prime} & -m_{1} m_{1}^{\prime} \\
1 & m_{2} & -m_{2}^{\prime} & -m_{2} m_{2}^{\prime} \\
1 & m_{3} & -m_{3}^{\prime} & -m_{3} m_{3}^{\prime}
\end{array}\right\|,
$$

* That is, three families of the web are regular at those of their respective lines which contain points of $\Gamma_{1}$. 
is of rank three for each $(x, y)$. For, if we assume the contrary, we have

$$
\left|\begin{array}{ccc}
1 & m_{1} & m_{1}^{\prime} \\
1 & m_{2} & m_{2}^{\prime} \\
1 & m_{3} & m_{3}^{\prime}
\end{array}\right|=\left|\begin{array}{ccc}
1 & m_{1} & m_{1} m_{1}^{\prime} \\
1 & m_{2} & m_{2} m_{2}^{\prime} \\
1 & m_{3} & m_{3} m_{3}^{\prime}
\end{array}\right|=0 \text {, }
$$

which yields upon eliminating $m_{1}^{\prime}$

$$
\left(m_{1}-m_{2}\right)\left(m_{1}-m_{3}\right)\left(m_{2}^{\prime}-m_{3}^{\prime}\right)=0 .
$$

But this is impossible, since there are exactly four lines at each point of a 4-web. Thus (2) is a set of three linear homogeneous equations of rank three in four quantities $p_{j}$. Therefore, all solutions may be put in the form

$$
\lambda L_{j}
$$$$
(j=1,2,3,4),
$$

where $\lambda$ is a parameter, and the $L$ 's, which are certain minor determinants in the matrix (3), are polynomials in the $m$ 's and $m^{\prime \prime}$. For each $(x, y)$ in $V$, the $p$ 's are uniquely determined, and are solutions of (2). Hence for each $(x, y)$ in $V$, there is a $\lambda(x, y)$ such that

$$
p_{j}=\lambda(x, y) L_{j} \quad(j=1,2,3,4),
$$

where $\lambda(x, y)$ cannot vanish, since $J$ does not.

Thus, substituting for the $p$ 's in (1), we have

$$
\frac{d y^{\prime}}{d x^{\prime}}=\frac{L_{1}+L_{2}(d y / d x)}{L_{3}+L_{4}(d y / d x)}
$$

and

$$
K=\left|\begin{array}{ll}
L_{1} & L_{2} \\
L_{3} & L_{4}
\end{array}\right| \neq 0
$$

where the $L$ 's are differentiable functions of $x$ and $y$. Therefore we may extend the transformation once more. We obtain

$$
\frac{d^{2} y^{\prime}}{d x^{\prime 2}}=\frac{\alpha+\beta \frac{d y}{d x}+\gamma\left(\frac{d y}{d x}\right)^{2}+\delta\left(\frac{d y}{d x}\right)^{3}+K \frac{d^{2} y}{d x^{2}}}{\lambda\left(L_{3}+L_{4} \frac{d y}{d x}\right)^{3}} .
$$

Since four non-vertical lines through each point of $V$ go into non-vertical lines, there are at each point of $V$ four directions at which $d^{2} y^{\prime} / d x^{\prime 2}$ vanishes with $d^{2} y / d x^{2}$. Thus the equation in $m$

$$
\alpha+\beta m+\gamma m^{2}+\delta m^{3}=0
$$

* We are following the method used by Kasner, loc. cit. 
has four roots for each $(x, y)$ and

$$
\alpha=\beta=\gamma=\delta=0 .
$$

Therefore, we have from (5)

$$
\frac{d^{2} y^{\prime}}{d x^{\prime 2}}=\frac{K \frac{d^{2} y}{d x^{2}}}{\lambda\left(L_{3}+L_{4} \frac{d y}{d x}\right)^{3}} .
$$

Thus, if at a point of $V, d y / d x$ has a value such that $L_{3}+L_{4}(d y / d x)$ is not zero, then for this value, $d^{2} y^{\prime} / d x^{\prime 2}$ vanishes with $d^{2} y / d x^{2}$.

Now consider $l$, any non-vertical line interval in $V$. If $L_{3}+L_{4}(d y / d x)$ vanishes identically on $l$, its image $l^{\prime}$ is a vertical interval. Suppose that $L_{3}+L_{4}(d y / d x)$ does not vanish at $A$, a point on $l$. Then, since $L_{3}$ and $L_{4}$ are continuous, there is an interval on $l$, containing $A$, at each point of which $L_{3}+L_{4}(d y / d x)$ is not zero. If $L_{3}+L_{4}(d y / d x)$ equals zero anywhere on $l$, there is, on $l$, a point $B$ such that this expression vanishes at $B$, but not on the segment $A B$. Thus, on the image of segment $A B, d^{2} y^{\prime} / d x^{\prime 2}$ is zero and $d y^{\prime} / d x^{\prime}$ is a finite constant. But $d y^{\prime} / d x^{\prime}$ is infinite at $B^{\prime}$, the image of $B$, which is absurd since the $L$ 's in (4) are continuous. Thus it is impossible that $L_{3}+L_{4}(d y / d x)$ vanish on $l$. Hence $d^{2} y^{\prime} / d x^{\prime 2}$ is zero everywhere on $l^{\prime}$, and $l^{\prime}$ is a line interval.

We have thus shown that all non-vertical line intervals in $V$ go into line intervals. This implies that vertical intervals, also, go into line intervals. Thus (4) defines a projectivity on each pencil of lines whose vertex is in $V$, and it easily follows that the transformation is projective on $V$. This completes the proof of the theorem under the original supposition.

Now let us suppose that in no sub-region of $\Gamma$ are three families of the given web regular. Then there are at least two families of the web which are not regular in any neighborhood of a given point of $\Gamma$.

Let $N$ be a point of $\Gamma$, and $f$ and $g$ two families of the web which are not regular in any neighborhood of $N$. Let $h$ and $k$ be the other two families of the web. Then in each neighborhood of $N$, there is a line of $f$ and a line of $g$ at which $f$ and $g$ respectively are not regular. Hence in a sufficiently small neighborhood of $N$, we can find a line $f_{1}$, at which $f$ is not regular, such that $f_{1}$ intersects $g_{N}$. Similarly, we can find a line $g_{1}$ at which $g$ is not regular, such that $g_{1}$ intersects $f_{1}$. Let $f_{1}$ and $g_{1}$ intersect at $Q$.

We can easily prove that the transformation is projective in the neighborhood of $Q$. Let $C, D$ be two points on $f_{1}$ distinct from $Q$, and $E, F$ two points 
on $g_{1}$, also distinct from $Q$. Consider $S$, the projective transformation which carries $C, D, E, F$ into their respective images under the given transformation. The given transformation and $S$ coincide at $C, D, E, F, Q$. By Lemma $\mathrm{XI}$, the transformation is a projectivity on $f_{1}$ and $g_{1}$. Hence the transformation and $S$ are identical on $f_{1}$ and $g_{1}$, by the fundamental theorem of projective geometry. Let $W$ be a neighborhood of $Q$, such that if $X$ is in $W, h_{X}$ and $k_{X}$ intersect both $f_{1}$ and $g_{1}$. Then, if $X$ is in $W$ and not on $h_{Q}$ or $k_{Q}$, the images of $h_{X}$ under the given transformation and $S$ are sub-sets of the same infinite line. And the same is true for $k_{X}$. Thus the transformations are identical for all such points $X$. Since they are continuous, they coincide for all points of $W$. Thus, the transformation is projective on $W$, which establishes the theorem.

Definition. A family of lines is called a pencil if its lines concur when extended.* The point of concurrence is the vertex of the pencil.

Now we may prove the following analogue of Theorem I, for a region of the euclidean plane.

ThEOREM VI. A one-to-one transformation of region $\Gamma$ is projective, if it carries a line and a web of three independent pencils in $\Gamma$ into a line and a web of three pencils, respectively.

Let $T$ be the transformation and $\pi, \pi^{\prime}$ the planes which contain the given and image webs respectively. Let $f$ and $g$ be two pencils of the given web. Let $S_{1}$ be a projective transformation on $\pi$ which carries $f$ and $g$ into parallel pencils. Since the vanishing line of $S_{1}$, if it exists, contains no point of $\Gamma, S_{1}$ is a topological transformation over $\Gamma$. Thus $S_{1}$ carries $\Gamma$ into a region $\Gamma_{1}$, and the given web into a web, in $\Gamma_{1}$, consisting of two parallel pencils and an ordinary pencil.

In the same way, we apply to $\pi^{\prime}$ a projective transformation $S_{2}$, which carries the images of $f$ and $g$ under $T$ into parallel pencils. Thus $R$, the resultant of $S_{1}^{-1}, T$ and $S_{2}$, is a one-to-one transformation of $\Gamma_{1}$, which carries a web composed of two parallel pencils $f, g$ and an ordinary pencil $h$ into a web composed of two parallel pencils $p, q$ and a third pencil $r$. $R$ also carries an additional line $l$ into a line $l^{\prime}$. We shall prove the theorem by showing that $R$ is projective in the neighborhood of a point.

Set up a cartesian coordinate system in $\pi$, with the vertex of pencil $h$ as origin, and with coordinate axes parallel to lines of pencils $f, g$ respectively. Let $A$, any point on $l$, have coordinates $(1,1)$. Similarly, establish a coordinate system in $\pi^{\prime}$ so that the point with coordinates $(1,1)$ is $D$, the image of $A$ under $R$, and the origin is an arbitrary point on the infinite line which

* We make the usual agreements about "ideal points" so that our results may apply to parallel pencils. 
contains $r_{D}$. Choose the coordinate axes parallel to lines of pencils $p, q$ respectively.

Let $(x, y)$ represent any point of $\Gamma_{1}$, and $\left(x^{\prime}, y^{\prime}\right)$, its image. Then $R$ carries line $y=x$ and pencils $x=t, y=t$ into $y^{\prime}=x^{\prime}$ and $x^{\prime}=t^{\prime}, y^{\prime}=t^{\prime}$ respectively. It follows that, in the neighborhood of $(1,1), R$ can be represented by the equations

$$
x^{\prime}=\phi(x), \quad y^{\prime}=\phi(y),
$$

where $\phi(1)=1$.

Let $y=a x+1-a, y^{\prime}=b x^{\prime}+1-b$ be the equations of $l, l^{\prime}$ respectively. Then we have

$$
\phi(a x+1-a)=b \phi(x)+1-b
$$

for all values of $x$ sufficiently close to 1 .

Now we shall show that $r$ is an ordinary pencil. Suppose that it is not. Then since $r$ contains the line $y^{\prime}=x^{\prime}$, it can be represented by $y^{\prime}=x^{\prime}+t$. Thus, since $h$ is of the form $y=m x$, we have

$$
\phi(m x)=\phi(x)+p(m)
$$

for all $x$ and $m$ such that $(x, m x)$ is in the neighborhood of $(1,1)$. It follows that (3) holds for all $x$ and $m$ in the neighborhood of 1, i.e., for all $x$ and $m$ between $1-\epsilon$ and $1+\epsilon$, where $\epsilon$ is a real number.

In (3) we assign to $x$ the value 1 , and easily derive

$$
\phi(m x)=\phi(m)+\phi(x)-1 .
$$

Applying (4) to (2) we get

$$
\begin{aligned}
\phi[a m x+(1-a) m] & =\phi(m)+b \phi(x)-b \\
& =b[\phi(m)+\phi(x)]+(1-b) \phi(m)-b .
\end{aligned}
$$

Thus

$$
\phi[a m x+(1-a) m]=b \phi(m x)+(1-b) \phi(m)
$$

holds for all $m$ and $x$ in the neighborhood of 1 . If in (5) we replace $m x$ by $x$, and $m$ by $y$, we may assert

$$
\phi[a x+(1-a) y]=b \phi(x)+(1-b) \phi(y)
$$

for all $x$ and $y$ in the neighborhood of 1 .

If we substitute 1 for $x$ in (6), then 1 for $y$ in (6), and eliminate $b \phi(x)$ and $(1-b) \phi(y)$ between the resulting equations and (6), we get

$$
\phi[a x+(1-a) y]=\phi(a x+1-a)+\phi[a+(1-a) y]-1,
$$


and hence

$$
\begin{aligned}
& \phi[(a x+1-a)+\{a+(1-a) y\}-1] \\
& =\phi(a x+1-a)+\phi[a+(1-a) y]-1 .
\end{aligned}
$$

Thus

$$
\phi(u+v-1)=\phi(u)+\phi(v)-1
$$

holds for all $u, v$ in the neighborhood of 1 . Expressing (4) with $u$ and $v$ as variables, we have in view of (7)

$$
\phi(u v)=\phi(u+v-1) .
$$

Substituting $1 / u$ for $v$ in (8), we have

$$
\phi[u+(1 / u)-1]=\phi(1)
$$

for $u$ close to 1 . This contradicts the fact that $R$ is a one-to-one transformation. Thus the assumption that $r$ is not an ordinary pencil is false.

In the discussion thus far, the origin of coordinates in plane $\pi^{\prime}$ has not been uniquely determined. Now we choose the vertex of the pencil $r$ as the origin in $\pi^{\prime}$. Relations (1) and (2) hold as before, and in addition, since $y=m x$ goes into $y^{\prime}=m^{\prime} x^{\prime}$, we have

$$
\phi(m x)=\phi(m) \phi(x)
$$

for $m$ and $x$ in the neighborhood of 1 .

Applying (9) to (2), we have

$$
\phi[a m x+(1-a) m]=b \phi(m x)+(1-b) \phi(m)
$$

for $m$ and $x$ near 1. But (10) is identical with (5). Thus (7), which follows directly from (5), holds for all $u, v$ near 1 .

For convenience, we write (9) as

$$
\phi(u v)=\phi(u) \phi(v) .
$$

The result follows from the functional equations (7) and (11). We apply to these equations the substitution

$$
u=1+U, \quad v=1+V,
$$

and change the function from $\phi$ to $\psi$ where

$$
\psi(Z)=\phi(1+Z)-1 .
$$

As a result, we have for $U, V$ near zero

$$
\psi(U+V)=\psi(U)+\psi(V)
$$


and

$$
\psi(U V+U+V)=\psi(U) \psi(V)+\psi(U)+\psi(V) .
$$

Applying (12) to (13), we have

$$
\psi(U V)=\psi(U) \psi(V) .
$$

Relations (12) and (14) enable us to show that $\psi(x)=x$ in the neighborhood of zero. The method is essentially the same as that used in the corollary to Theorem I, where (12) and (14) hold with $U$ and $V$ as unrestricted real variables. Thus $\phi(x)=x$ in the neighborhood of $x=1$, and transformation $R$ is projective in the neighborhood of $(1,1)$, from which the desired result follows by Theorem IV.

The following theorem and corollary are concerned with the case of three dependent pencils.

THEOREM VII. A one-to-one transformation of a region $\Gamma$, which is not the entire euclidean plane, is affine, if it carries three parallel pencils in $\Gamma$ into three parallel pencils.

Let $f, g, h$ be the three pencils contained in $\Gamma$, and $p, q, r$ their respective images under the given transformation $T$. Let $\Gamma^{\prime}$ be the image of $\Gamma$, and $\pi, \pi^{\prime}$ the planes which contain $\Gamma, \Gamma^{\prime}$, respectively.

Since $\Gamma$ is a proper sub-set of the euclidean plane, one of the pencils $f, g, h$ contains a line which is not an infinite line, i.e., it contains a half line or a finite interval. Let us suppose that $f_{A}$ is such a line. Let $D$ be the image of $A$ under $T$.

We shall show that the inverse transformation $T^{-1}$ is projective in the neighborhood of $D$. We establish cartesian coordinate systems in $\pi$ and $\pi^{\prime}$, locating the respective origins at $A$ and $D$, and the points $(1,1)$ on $h_{A}, r_{D}$ respectively, and choosing the coordinate axes from the pencils $f, g, p, q$ respectively.

It follows, by the method used in the preceding theorem, that $T^{-1}$ may be represented in the neighborhood of $D$ by

$$
x=\phi(u), \quad y=\phi(v),
$$

where

$$
\phi(u+v)=\phi(u)+\phi(v)
$$

for all $u$ and $v$ near zero. Moreover, the fact that $T^{-1}$ carries $p_{D}$ into a half line or a finite interval implies that $\phi(u)$ has an upper or lower bound. From 
this we can show that $\phi(u)=c u$ in the neighborhood of zero, where $c$ is a constant.*

Hence $T^{-1}$ is projective, by Theorem IV. It obviously is affine since (1) defines an affinity in the neighborhood of $(0,0)$. Thus $T$ is affine also.

Corollary. A one-to-one transformation of a region $\Gamma$, which is not the entire euclidean plane, is projective, if it carries a web of three dependent pencils in $\Gamma$ into a similar web.

By the application of projective transformations to the given and image figures, we can reduce the given transformation to one which carries three parallel pencils into three parallel pencils. Thus the resultant transformation is affine, and the original is projective.

It is evident that the condition in the above theorem and corollary, that $\Gamma$ be a proper sub-set of the euclidean plane, may be dispensed with, if we assume that the transformation is topological.

Finally, we prove the following theorem.

THEOREM VIII. A one-to-one transformation of a region is projective, if it carries a 3-web of lines composed of two pencils and a family not a pencil, into a similar 3-web, families of the same type corresponding. $\dagger$

By the application of projective transformations as in Theorem VI, the given transformation can be reduced to a transformation $T$, which carries a web of lines composed of two parallel pencils $f, g$ and a family $h$, not a pencil, into a similar web. It suffices to show that $T$ is projective in the neighborhood of a point.

Let $\Gamma$ be the region containing $f, g, h$ and let $A$ be any point of $\Gamma$. If there exists a positive number $\epsilon$ such that all the lines of $h$ whose distance to $A$ is less than $\epsilon$ concur at $B$, when extended, we shall say that $h$ is a pencil with vertex $B$ in the neighborhood of $A$.

We shall show that there exists a point $M$ in $\Gamma$, such that $h$ is not a pencil in the neighborhood of $M$. Suppose that this is not so. Then $h$ is a pencil in the neighborhood of each point of $\Gamma$, and we shall prove that $h$ is a pencil. Let $C$ be a fixed point of $\Gamma$, and $X$, a variable point of $\Gamma$, distinct from $C$. Let $h$ be a pencil with vertex $D$, in the neighborhood of $C$. We shall show that $h_{X}$, when extended, contains $D$.

* This can be proved, essentially, by the method which Darboux (loc. cit., pp. 56, 57) employs to derive the analogous result when (2) holds for all real $u, v$.

$\dagger$ Dubourdieu, Abhandlungen Hamburg Seminar, vol. 7 (1929), p. 219, derives this result on the assumption that the transformation and the families are differentiable. Kasner, in his studies of nearcollineations, also has derived a related result. See Bulletin of the American Mathematical Society, vol. 36 (1930), p. 796, abstract No. 388. 
Let $l$ be a broken line joining $C$ and $X$ in $\Gamma$. Separate the points of $l$ into two sets, the first of which contains all points $P$ such that for each $Q$ on $l$ between $C$ and $P, h_{Q}$, when extended, passes through $D$. Let the second set contain all other points of $l$. If the second set is not empty, there is a point $E$ on $l$ such that we can find two lines of $h$ as close to $E$ as we please, one and only one of which passes through $D$, when extended. But this is impossible since $h$ is a pencil in the neighborhood of $E$. Thus the second set is empty, and $h_{X}$, when extended, passes through $D$. Therefore, $h$ is a pencil since $X$ is an arbitrary point of $\Gamma$. This absurdity implies the existence of a point $M$, such that $h$ is not a pencil in the neighborhood of $M$.

Now establish a cartesian coordinate system in the given plane with $M$ as the origin, $N$ any other point on $h_{M}$ as $(1,1)$, and $f_{M}, g_{M}$ as the coordinate axes. In the image plane introduce coordinates similarly, with the images of $M, N$ as $(0,0),(1,1)$, respectively, and the images of $f_{M}, g_{M}$ as coordinate axes. As in previous theorems, $T$ may be represented, in the neighborhood of $M$, by

$$
x^{\prime}=\phi(x), \quad y^{\prime}=\phi(y) .
$$

In view of Lemma $\mathrm{I}$, the lines of $h$ which are sufficiently close to $M$ are represented by

$$
y=a x+b
$$

where $\delta_{1}<b<\delta_{2}, \delta_{1}<0<\delta_{2}, a=a(b)$ and $a(0)=1$. It follows from Lemma III that $a(b)$ is a continuous function.

Since each line of $h$ goes into a non-vertical straight line,

$$
\phi(a x+b)=c \phi(x)+d
$$

holds for all $x$ and $b$ near zero, where $c=c(b)$ and $d=d(b)$. If $b_{1}$ is an arbitrary value of $b$, we may similarly assert

$$
\phi\left(a_{1} x+b_{1}\right)=c_{1} \phi(x)+d_{1},
$$

where $a_{1}=a\left(b_{1}\right), c_{1}=c\left(b_{1}\right), d_{1}=d\left(b_{1}\right)$. Substituting $a_{1} x+b$ for $x$ in (1) and $a x+b$ for $x$ in (2), we have, for $x, b, b_{1}$ sufficiently close to zero,

$$
\phi\left(a a_{1} x+a b_{1}+b\right)=c c_{1} \phi(x)+c d_{1}+d
$$

and

$$
\phi\left(a a_{1} x+a_{1} b+b_{1}\right)=c c_{1} \phi(x)+c_{1} d+d_{1} .
$$

Eliminating $\phi(x)$ between (3) and (4), we have

$$
\phi\left(a a_{1} x+a b_{1}+b\right)=\phi\left(a a_{1} x+a_{1} b+b_{1}\right)+c d_{1}+d-c_{1} d-d_{1} .
$$


Substituting $\left(u-a_{1} b-b_{1}\right) /\left(a a_{1}\right)$ for $x$ in (5), we have

$$
\phi\left(u+a b_{1}-a_{1} b+b-b_{1}\right)=\phi(u)+c d_{1}+d-c_{1} d-d_{1},
$$

for all $u, b, b_{1}$ sufficiently close to zero. Letting $u$ be zero in (6), we easily derive

$$
\phi\left[u+p\left(b, b_{1}\right)\right]=\phi(u)+\phi\left[p\left(b, b_{1}\right)\right]
$$

where

$$
p\left(b, b_{1}\right)=a b_{1}-a_{1} b+b-b_{1} .
$$

The function $p$ is continuous and $p(0,0)=0$. Thus in the neighborhood of $(0,0)$ either $p$ is identically zero, or $p$ assumes all values in an interval which terminates at zero. The former is impossible since it implies that

$$
p\left(b, b_{1}\right) \equiv\left|\begin{array}{lll}
1 & 1 & 0 \\
1 & a & b \\
1 & a_{1} & b_{1}
\end{array}\right|=0
$$

for all $b, b_{1}$ near zero. And the vanishing of the determinant in (8) is a sufficient condition that the lines $y=x, y=a x+b, y=a_{1} x+b_{1}$ concur. Thus family $h$ is a pencil in the neighborhood of $M$, which is absurd. Therefore $p\left(b, b_{1}\right)$ is not identically zero in any neighborhood of $(0,0)$, and assumes all values on an interval terminating at zero, in such a neighborhood.

Hence, if we substitute $v$ for $p\left(b, b_{1}\right)$ in (7) we may assert that

$$
\phi(u+v)=\phi(u)+\phi(v)
$$

holds for all $u, v$ on the respective intervals $(-\delta, \delta),(0, \delta)$, where $\delta$ is a real number sufficiently close to zero. Letting $v=-u$ in (9) we have

$$
\phi(-u)=-\phi(u)
$$

for $u$ on the interval $(0,-\delta)$. Then if $u$ and $v$ are on $(-\delta / 2, \delta / 2)$ and $(0,-\delta / 2)$ respectively, we have

$$
\phi[u+v+(-v)]=\phi(u+v)-\phi(v),
$$

and

$$
\phi(u+v)=\phi(u)+\phi(v) .
$$

Thus (9) holds for all $u$ and $v$ sufficiently close to zero.

From (1) we easily derive

$$
\phi[a(b) x+b]=c(b) \phi(x)+\phi(b) .
$$

From (9) and (10) we get 


$$
\phi[a(b) x]=c(b) \phi(x) .
$$

The function $a(b)$ is continuous, is not constant in the neighborhood of zero, and $a(0)=1$. Thus, if we substitute $t$ for $a(b)$ in (11), we may assert that

$$
\phi(t x)=\psi(t) \phi(x)
$$

holds for all $x$ and $t$ on the respective intervals $(-\eta, \eta)$ and $(1,1+\eta)$. Substituting $1+z$ for $t$ in (12), we have

$$
\phi(x+x z)=\phi(x) \psi(1+z)
$$

for $x$ and $z$ on $(-\eta, \eta)$ and $(0, \eta)$ respectively. Substituting $z$ for $x$ in (13) and eliminating $\psi(1+z)$, we have

$$
\phi(x+x z)=\frac{\phi(x) \phi\left(z+z^{2}\right)}{\phi^{2}(z)}
$$

and hence, by (9), we have

$$
\phi(x z)=\frac{\phi(x) \phi\left(z^{2}\right)}{\phi(z)} .
$$

Interchanging $x$ and $z$ in (14) and eliminating $\phi(x z)$, we have

and

$$
\phi\left(x^{2}\right)=\frac{\phi^{2}(x) \phi\left(z^{2}\right)}{\phi^{2}(z)}
$$

$$
\phi\left(x^{2}\right)=k \phi^{2}(x)
$$

for $x$ on $(0, \eta)$.

By an argument similar to that of the corollary to Theorem I, we can show from relations (9) and (15) that $\phi(x)=x / k$ in the neighborhood of zero. Thus $T$ is projective in the neighborhood of $(0,0)$ and the proof is complete.

BrookLYN College, BROOKIYN, N. Y. 